Portland State University

PDXScholar

1988

\title{
Two dimensional and three dimensional path planning in robotics
}

Hyun Suk Kim

Portland State University

Follow this and additional works at: https://pdxscholar.library.pdx.edu/open_access_etds

Part of the Electrical and Computer Engineering Commons, and the Robotics Commons Let us know how access to this document benefits you.

\section{Recommended Citation}

Kim, Hyun Suk, "Two dimensional and three dimensional path planning in robotics" (1988). Dissertations and Theses. Paper 3814.

https://doi.org/10.15760/etd.5698

This Thesis is brought to you for free and open access. It has been accepted for inclusion in Dissertations and Theses by an authorized administrator of PDXScholar. Please contact us if we can make this document more accessible: pdxscholar@pdx.edu. 
AN ABSTRACT OF THESIS OF Hyun Suk Kim for the Master of Science in Electrical Engineering presented March 10, 1988.

Title: $\quad$ Two Dimensional and Three Dimensional Path Planning in Robotics. APPROVED BY MEMBERS OF THE THESIS COMMITTEE:
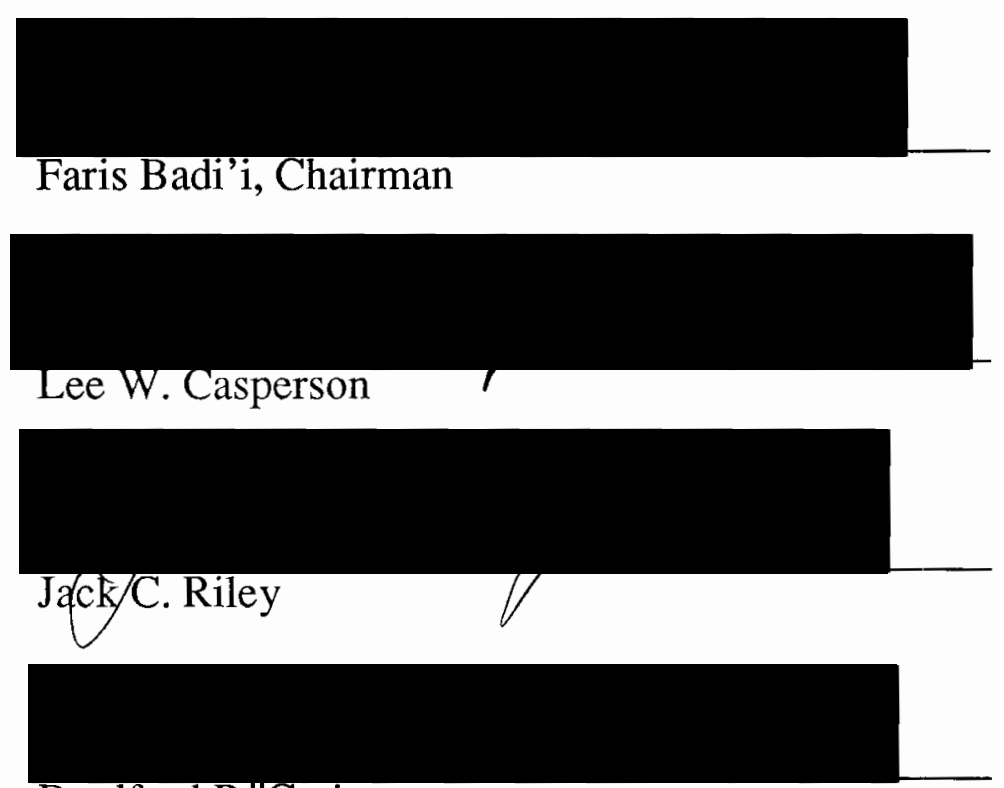

Bradford R.VCrain

A methodology for 2D and 3D collision free path planning algorithm in a structured environment is presented. The isolated free convex areas are represented as a nodes in a graph, and a graph traversal strategy that dynamically allocates costs to graph path is used. Modification of the algorithm for small computational time and optimality is discussed. The $3 \mathrm{D}$ path planning is done in the three orthogonal two-dimensional projections of a $3 \mathrm{D}$ environment. Collision checking to increase the optimality for $3 \mathrm{D}$ paths is done in each of the three orthogonal two-dimensional subspaces. 


\title{
TWO DIMENSIONAL AND THREE DIMENSIONAL PATH PLANNING IN ROBOTICS
}

\author{
by \\ HYUN SUK KIM
}

A thesis submitted in partial fulfillment of the requirements for the degree of

\author{
MASTER OF SCIENCE \\ in \\ ELECTRICAL ENGINEERING
}

Portland State University

1988 
TO THE OFFICE OF GRADUATE STUDIES:

The members of the Committee approve the thesis of Hyun Suk Kim presented March 10, 1988.

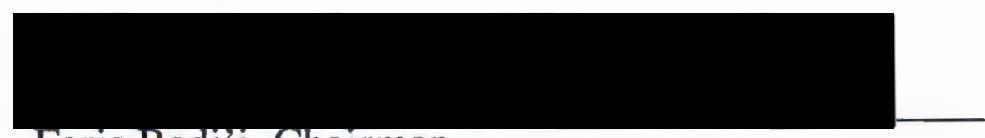

Faris Badi' 1, Chairman
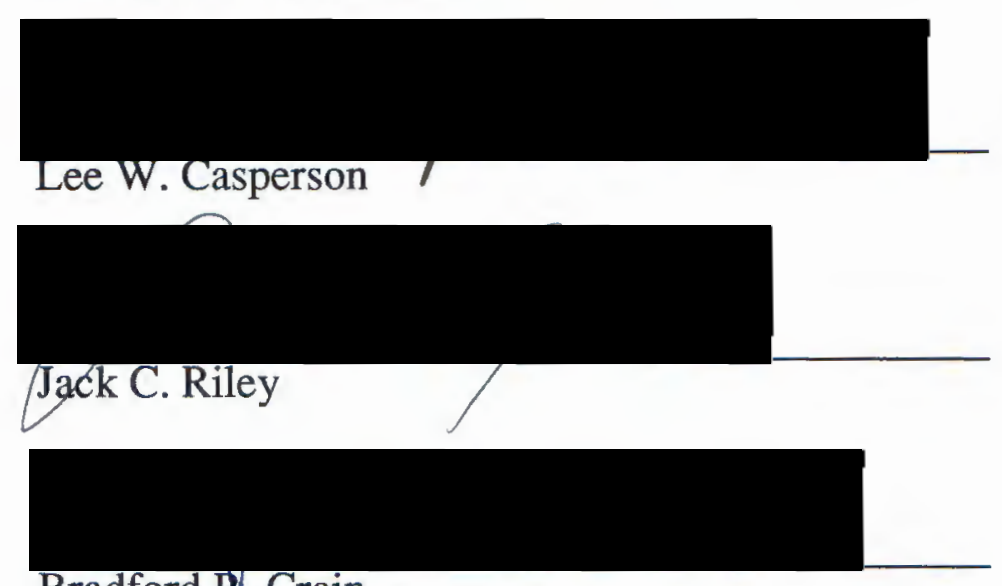

APPROVED:

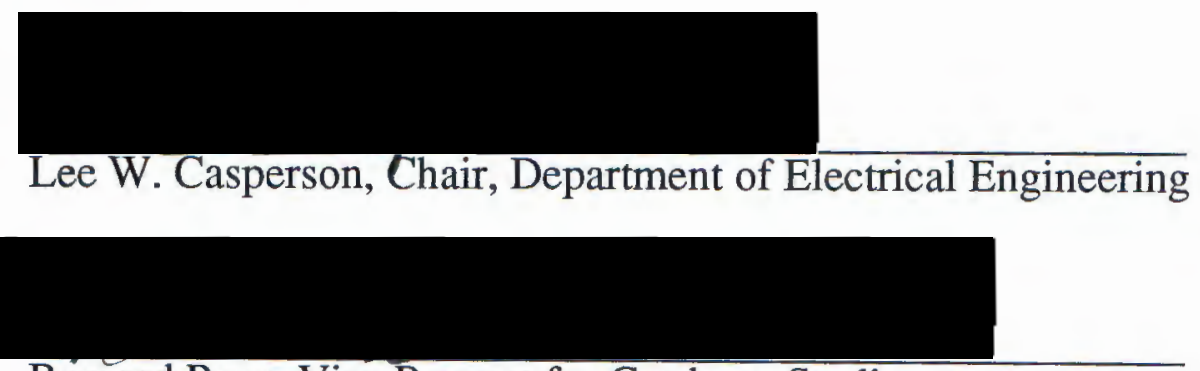

Bernard Ross, Vice Provost for Graduate Studies 


\section{TABLE OF CONTENTS}

PAGE

TABLE OF CONTENTS …...................................................................

LIST OF TABLES ……………………………………........................ v

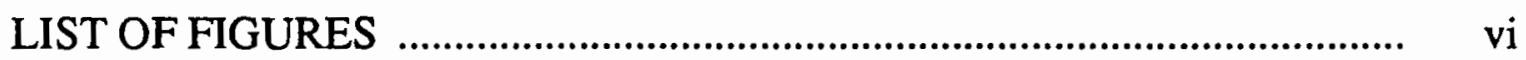

CHAPTER

I INTRODUCTION ....................................................................

II TWO DIMENSIONAL PATH PLANNING …………………………... 6

Convex Areas And Obstacle Areas .................................................. 6

Isolation of Prime Convex Area

Finding Obstacle Areas

Setting Up The Graph ................................................................ 10

Path Planning ……................................................................... 12

Traversing

Backtracking

Analysis And Modifications .......................................................... 21

Time Complexity

III THREE DIMENSIONAL PATH PLANNING .................................... 35

Three Orthogonal Space Projections ................................................. $\quad 35$ 
Path Planning

Obtaining a Three-dimensional Path

Modifications

Analysis And Simulation Of The Algorithm

40 Time Complexity

IV CONCLUSION

\section{APPENDIX}

A SAMPLE LAYOUT …......................................................... 55

B TWO DIMENSIONAL PATH PLANNING PROGRAM ................. 59

C THREE DIMENSIONAL PATH PLANNING PROGRAM ............... 64 


\section{LIST OF TABLES}

TABLE

PAGE

2-I Comparision Of Path Lengths And Computational Time

Obtained By $\beta=1$ And $\beta=2$ With Those Obtained

By Modified Method ................................................................. 28

2-II Path Length And Computational Time Obtained by Using

Modified Method

29

2-III Relationship Between Graph Parameters And Path

Computational Time 


\section{LIST OF FIGURES}

FIGURE

PAGE

2-1 Typical Layout Of Three Obstacle .............................................. 7

2-2 The Procedure To Obtain All Prime Convex Areas ...................... 11

2-3 Obtaining All Obstacle Areas .................................................... 12

2-4 Graph Of Intersecting Prime Convex Areas And Areas Of

Intersection Associated With Each Arc. .................................. 13

2-5 Development Of Path Segment Based Upon Relative Position

Of A Future Node .................................................................... 15

2-6 Traversal And Backtracking Procedure And Deliting Links

From Graph ....................................................................... 18

2-7 Sample Layout Resulted From A Highly Restricted Search ........... 23

2-8 Sample Layout Resulted From The Modification 1 Which Is

Applied Once ....................................................................... 25

2-9 Sample Layout With 4 Obstacles And Sample Paths Obtained

By Procedure Of This Paper For $\beta=1$ And Modified .............. 26

2-10 Sample Cluttered Layout And Sample Paths ............................... 27

2-11 Sample Layout And Sample Paths ........................................... 33

3-1 The Difference Between An Unmodified Path And A

Modified Path ................................................................................ 44

3-2 Examples Of Three-dimensional Path Planning ............................. 45 
3-3 Example Of The Severe Occluding Effect ................................... 50

A-1 Typical Layout Of two obstacles .............................................. 56

A-2 The Procedure To Obtain All Prime Convex Areas ...................... 57

A-3 Graph Of Intersecting Prime Convex Areas ................................ 58 


\section{CHAPTER I}

\section{INTRODUCTION}

The task of a moving object among a set of obstacles is a two step process: 1) planning collision free paths 2 ) controlling the robot to execute the planned paths gracefully. This paper deals with the former issue by suggesting a path finding strategy that is computationally efficient and yields near-optimal results in terms of Euclidean distance (shortest paths).

Prior approaches to collision free path planning can be summarized like this: The problem of planning safe trajectories is analyzed and a solution to the problem proposed by Udupa(14). Collision detection is checking intersection of the trajectory envelope and obstacles which is approximated by polyhedra-planefaced objects. But the environment undergoes minor changes because it is assumed that drastic changes are infrequent compared to the total number of trajectories planned. And optimal plans are not needed.

Lozano-Perez $(6,7,8)$ also adopted polyhedra as the model. He used $V G R A P H$ (visibility graph) to set up configuration space that can be mapped into a graph of vertices (representing corners of obstacles). His algorithm involves growing the obstacles and shrinking the moving object to an arbitrary reference point on the moving object. The trajectory of this reference point is found by graph search, where the graph arcs are allocated fixed weight, and is made up of straight lines. But the path planning that require almost continuous changes of orientation as well as position results in many transition obstacle set and is therefore expensive to compute. In Lozano-Perez's configuration space approach, each 
workspace may not always be possible with a finite views. This is due to occluding effects and insufficient information obtained from two-dimensional projections. Even for those obstacles whose recognitions or reconstructions are possible, the recognition or reconstruction processes are often computationally intensive.

In the approach used in this paper, the recognition or reconstruction of three-dimensional models for the obstacles in the workspace is not desirable. Rather, where there are obstacles and where there is free space in the threedimensional workspace are concerned. It is proposed to model the environment by a set of three orthogonal two-dimensional projections of the three-dimensional workspace. Three-dimensional collision checking is decomposed into the problem of two-dimensional collision checking in the three orthogonal two-dimensional projections. In the approach discussed in this paper, the small data input is used to represent the three orthogonal two-dimensional projections in place of visual input.

The path planning algorithm discussed in this paper drives benefit from the concept of convexity by identifying all the largest rectangular free areas. A graph is created with nodes corresponding to each such convex area and intersecting convex shapes are presented as adjacent nodes in each two-dimensional projection. Path planning is then reduced to finding a route from a source node to a destination node through the graph and choosing the best possible path based on a given cost function.

Several assumptions are made in this paper.

1) A circular moving object (a sphere for three-dimensional moving object) is assumed. This allows one to shrink the moving object to a reference point that is a center of a circle (or a sphere) and to grow the obstacles by the radius of the moving object in a two- 
dimensional projection. Therefore, if this reference point is collision free in any workspace, then the real moving object is also collision free. The reason for this assumption of a circular moving object is that one does not have to consider the moving objects with varing orientations.

2) Obstacles are approximated by iso-oriented rectange-plane-faced objects in which the edges are parallel to the coordinate axes. (The projected obstacles are rectangular shapes in two-dimensional projections.) This assumption helps in keeping the computational cost down.

3) The cost allocated to a path or a portion of a path is directly proportional to its geometric length.

There are several more factors such as computational time and how many turns a robot makes to determine optimality. Without any special cost function, however, it is very difficult to find relationship between turn and length. And the computational time is explained in following chapters. Chapter II presents the method that isolates all rectangular collision free areas. And two-dimensional path search through a node graph (concurrently geometric path search) and dynamic cost allocation procedure are developed. Because overlap of collision free areas is permitted, the graphs generated are little more complex than the ones used by previous researchers. The time complexity issues of the associated algorithm are also discussed in terms of inadmissible areas representing obstacles. In chapter III, the three-dimensional path planning is developed by extending the two-dimensional path planning algorithm to the three-dimensional space in the three orthogonal two-dimensional projections of the environment and computational results and time complexity are given. Chapter IV gives the conclusion. 


\section{CHAPTER II}

\section{TWO DIMENSIONAL}

\section{PATH PLANNING}

In this chapter, two dimensional path planning algorithm for a moving object using all the largest rectangular areas free of obstacles and obstacle areas is presented. The algorithm discussed in this paper is based on the Singh and Wagh's algorithm(13) of path planning. And this method is improved by eliminating the problems their algorithm has.

\section{CONVEX AREAS AND OBSTACLE AREAS}

The algorithm presented in this section tries to isolate the largest convex areas that are free of obstacles and find possible obstacle areas that are occupied by obstacles. In this paper, a assumption is made that obstacles are approximated by iso-oriented in which the obstacles might be overlapped each other. Therefore the exact shapes of obstacles are not concerned. This assumption helps in keeping the computational costs down. An algorithm used to find prime convex areas and obstacle areas is based on Quine-McClusky method(10).

A convex area is an area that is free of obstacles and has a property that any two point in that area can be joined by a straight line that lies entirely within that area. A prime convex area is a convex area and is not fully incorporated in any other single prime convex area. An obstacle area is an area that is occupied by inadmissible area representing obstacles. 


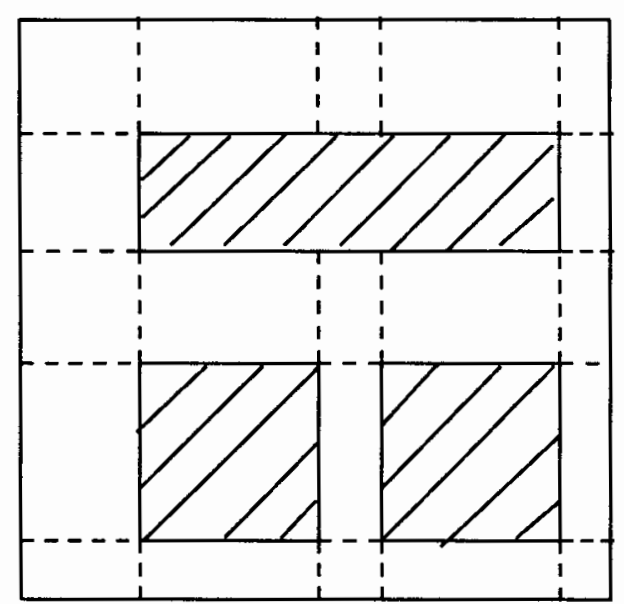

(a)

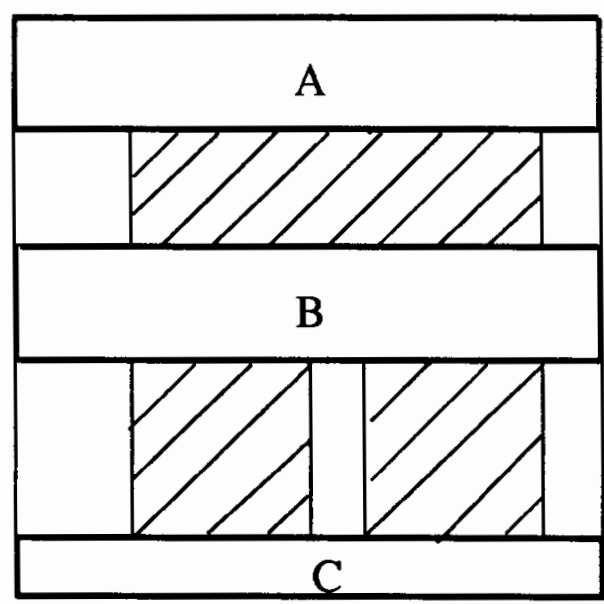

(b)

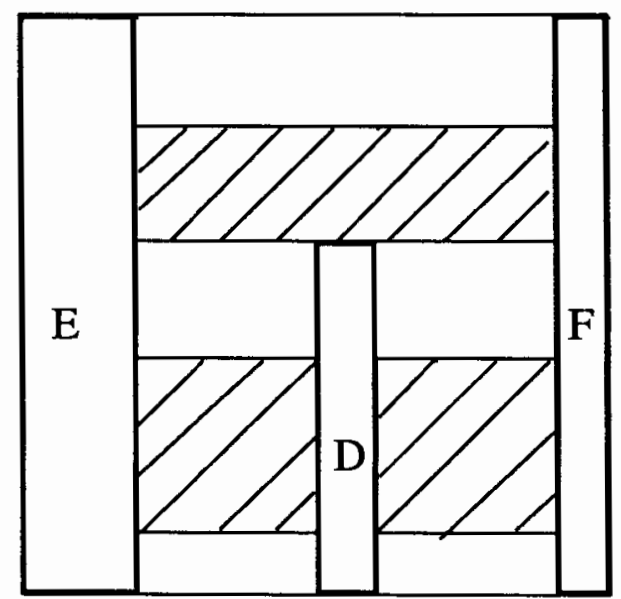

(c)

Figure 2-1. Typical layout of three obstacles. Layout is shown in (a) and the shaded areas marked A through $F$ in (b) and (c) represent all the prime convex areas in this layout.

Given a map of a model which is occupied by free areas and obstacles, an environment is partitioned by the edge of these obstacles into a grid of at most ( $2 n$ $+1) \times(2 n+1)$ rectangles where $n$ is the number of inadmissible areas representing obstacles. Each such rectangle is represented by a pair of binary strings each at most $2 n+1$ bit long. The left substring presents the relative $x$ position and the 
right the y position. For example, for $n=3$ as in figure 2-1(a), a partition that is the third from the left and the forth from the top could be represented by the string

\section{0}

A similar notation can be used for areas made up of several partitions. The string

\section{0}

represents a large rectangle made up of four partitions:

1000001000

0100001000

1000000100

0100000100

Isolation of Prime Convex Area. : The following algorithm may be used to identify all rectangular prime convex areas by fusing together free rectangular partitions from the grid described above. The rectangular areas that is free of obstacles are represented by 1 's and the rectangles occupied by obstacles are represented by 0 's.

Step 1

Represent each horizontal strip by means of a pair of binary strings each at most $2 n+1$ bit long. The left substring has those bits set which correspond to the free rectangles in the strip. The right substring has only one bit set corresponding to the vertical position of the strip. For example, the fourth strip of figure 2-1(a) is represented by 
Step 2

Find all the contiguous horizontal strips. This is done by breaking up the left substring into contiguous runs of 1 's and keeping the right substring unchanged in each part. If the left substring does not contains any 1's then the horizontal strip is checked off. This is useful in the process of finding obstacle areas. For example, the strip of step 1 can be broken into three contiguous horizontal strips:

1000000010

0010000010

0000100010

Step 3

Make a list of all strings generated by step 2 such that strings are grouped by the strips that generated them and the groups are ordered according to the vertical positions of the generating strips.

Step 4

Generate a new list of strings from the old list of strings based upon the following rules until the new list generated has only one group.

1) The new ith group of strings is generated by combining each string from the old $i$ th group with each string from the old $i+1$ th group, $\mathrm{i}=1,2, \ldots \ldots$

2) Two strings are combined by logically ORing the right substrings and logically ANDing the left substrings. If the new substring has a null (all zero) substring, discard that string. Otherwise, add it to the new list.

3) Every time a string is added to the new list, check off all the strings from the old lists that are covered by the new list. A string $S_{1}$ is said to be 
covered by a string $S_{2}$ if local ORing of two strings yields $S_{2}$.

A string from any list that is not checked off represents a prime convex area for the layout. The prime convex areas corresponding to the layout of figure $2-1$, produced by this procedure, are listed in figure $2-2$.

Finding Obstacle Areas. : The same algorithm is used to identify all rectangular obstacle areas. However, the rectangles occupied by obstacles are presented by 1 's and the free rectangles are represented by 0's. And if a string has a null left substring such as the first strip, the third strip, and the fifth strip of figure 2-1(a), check off that horizontal strip in the step 2 . The figure $2-3$ shows the procedure from step 1 through step 5 to obtain all obstacle areas.

\section{SETTING UP THE GRAPH}

The representation of information about the prime convex areas in generated in above section a usable data structure and setting up the node graph are explained in this section where each node represents a prime convex area. If there is a intersection between the areas of two nodes, they are joined by an arc and a node path segment exists i.e., the robot path planning involves traversing the graph from one node to another node if the areas they represent intersect each other and each arc has associated with it information about the geometrical intersection. Each arc is bi-directional. For example, if nodes $\mathrm{A}$ and $\mathrm{B}$ intersect each other, there are two directions of traversing the graph, A to B and B to A. Figure 2-4 shows such a graph obtained from the layout of figure 2-1(a) and also shows the areas of intersection associated with each arc.

Another sample layout with two obstacles is illustrated in Appendix A. The procedure of isolation of prime convex areas and the setting up the graph for 
list 1

1111110000

1000101000

1111100100

1010100010

1111100001

list 2

1111110000 (A)

$1000001000(\mathrm{X})$

0000101000 (X)

1111100100 (B)

$1000000010(\mathrm{X})$

$0010000010(\mathrm{X})$

0000100010 (X)

1111100001 (C)

list 4

$1000011100(\mathrm{X})$

$0000111100(\mathrm{X})$

$1000001110(X)$

0000101110 (X)

1000000111 (X)

0010000111 (D)

0000100111 (X) list 3

$1000011000(\mathrm{X})$

$0000111000(\mathrm{X})$

$1000001100(\mathrm{X})$

$0000101100(\mathrm{X})$

$1000000110(\mathrm{X})$

$0010000110(X)$

$0000100110(\mathrm{X})$

1000000011 (X)

$0010000011(\mathrm{X})$

0000100011 (X)

list 5

$1000011110(\mathrm{X})$

0000111110 (X)

$1000001111(\mathrm{X})$

$0000101111(\mathrm{X})$

list 6

$1000011111(\mathrm{E})$

$0000111111(\mathrm{~F})$

Figure 2-2. The procedure to obtain all prime convex areas for layout of figure 2-1(a). The prime convex areas remain unmarked and are labeled as A through $\mathrm{F}$.

that layout is presented in figure A-1 through figure A-3.

It should be noted here that the isolation of prime convex areas and obstacle areas, and setting up the graph needs to be done only once for a given environment and need not be repeated until it changes. For another layout, it can be done 


\section{list 1}

0000010000
0111001000
0000000100
0101000010
0000000001

list 2

$0000010000(\mathrm{X})$

$0111001000(\mathrm{~A})$

$0000000100(\mathrm{X})$

$0100000010(\mathrm{~B})$

$0001000010(\mathrm{C})$

$0000000001(\mathrm{X})$

Figure 2-3. Obtaining all obstacle areas for layout of figure 2-1(a). Obstacle areas are labeled as A through $\mathrm{C}$.

easily by changing simple input data. (It contains binary representation of layout and addresses of the edges of obstacles.)

\section{PATH PLANNING}

The basic strategy of robot path planning is traversing node graph from a node containing the source point to a node containing the destination point, and finding the optimal path in terms of graph nodes and a geometric representation of this path using a backtracking procedure.

If optimality is not a criterion, traversing the graph is straightforward. Starting with a node containing the source point (starting node, S), next node which can be reached by some collision-free path segment, can be found until a node containing the destination point (destination node, D) is reached. However, 


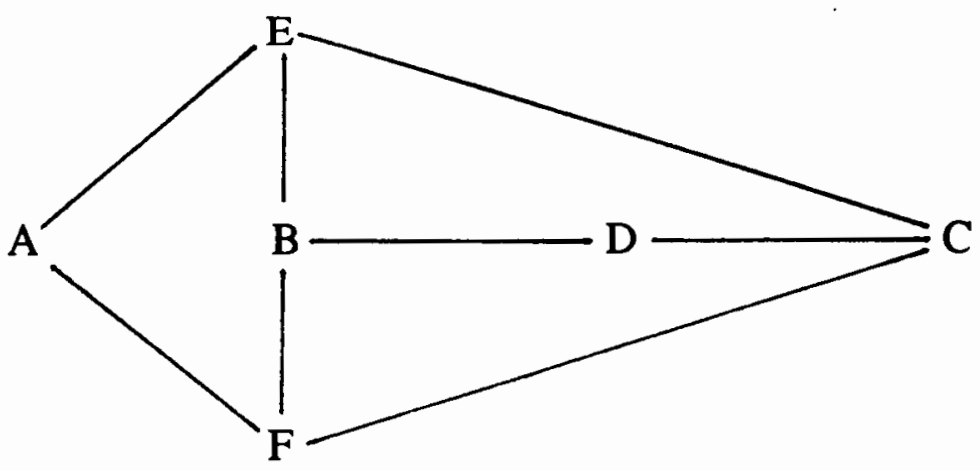

$\begin{array}{ccc}\text { arc } & \text { nodes } & \text { area of intersec } \\ \text { a } & \text { A - E } & 1000010000 \\ \text { b } & \text { A - F } & 0000110000 \\ \text { c } & \text { B - D } & 0010000100 \\ \text { d } & \text { B - E } & 1000000100 \\ \text { e } & \text { B - F } & 0000100001 \\ \text { f } & \text { C - D } & 0010000001 \\ \text { g } & \text { C - E } & 1000000001 \\ \text { h } & \text { C }- \text { F } & 0000100001\end{array}$

Figure 2-4. Graph of intersecting prime convex areas for layout of figure 2-1(a) and areas of intersection associated with each arc.

the consideration of optimality brings some complications.

1) Both the source and destination points may fall inside several different nodes since a point may belong to the intersection of several prime convex areas. Therefore all possible paths originating from valid starting nodes and terminating on valid destination nodes have to be considered.

2) Arcs can not have fixed weight (length cost) attached to them because any two points in one prime convex area are not necessarily equidistant to a point in another prime convex area. The cost of path segment from one node to another is dependent on where the path points are actually located in the prime convex areas and has to be computed each time an arc between two nodes exists. 
Therefore the basic algorithm is that all possible paths from start nodes to destination nodes have to be considered and that a path that has minimum cost in terms of geometric path representation is chosen by using a backtracking method.

Traversing : Moving from one node to another node where an arc exists between two nodes i.e., two prime convex areas intersect each other, is geometrically equivalent to choosing a point in the intersection of the two prime convex areas. However, the placement of this point within this intersection is dependent on where the path progress next. Thus a one node look ahead algorithm will be explained next.

Since graph arcs can not have fixed weights attached to them, the cost function must dynamically allocate costs to path segments as the path progress. Figure 2-5(a) shows graph node traversal from node $X_{i}$ to $X_{i+1}$ and then to either node $\mathrm{X}_{i+2}$ or $\mathrm{X}_{i+2}^{\prime}$. Figure 2-5(b) shows the development of the corresponding geometric representation of these path segments. Let $a, b$, and b' denote areas of intersection of $X_{i}$ and $X_{i+1}, X_{i+1}$ and $X_{i+2}$, and $X_{i+1}$ and $X_{i+2}^{\prime}$ respectively. And $\operatorname{mid}(b)$ and $\operatorname{mid}\left(b^{\prime}\right)$ denote the mid point of the two intersections $X_{i+1}$ and $X_{i+2}$, and $X_{i+1}$ and $X_{i+2}^{\prime}$ respectively. Assume that the current path point is $C_{i}$ in node $\mathrm{X}_{i}$.

Assume that the graph path progresses $X_{i} \rightarrow X_{i+1} \rightarrow X_{i+2}$. First, joint points $\mathrm{C}_{i}$ and $\operatorname{mid}(\mathrm{b})$ by a straight line. If the line intersect area $a$ which is intersection of node $X_{i}$ and node $X_{i+1}$, then the next path point $C_{i+1}$ is the point where the line first meets area $a$. The new point $C_{i+1}$ is in the node $X_{i+1}$. On the other hand, if the line does not intersect area $a$, then the next path point $\mathrm{C}_{i+1}^{\prime}$ is the corner of the area $a$ that is closest to the line. This second case is shown by the graph traversal $X_{i} \rightarrow X_{i+1} \rightarrow X_{i+2}^{\prime}$. If the current path point $C_{i}$ is on the boundary of intersection $a$, then the next path point is the same point $\left(C_{i}=C_{i+1}\right)$. The 


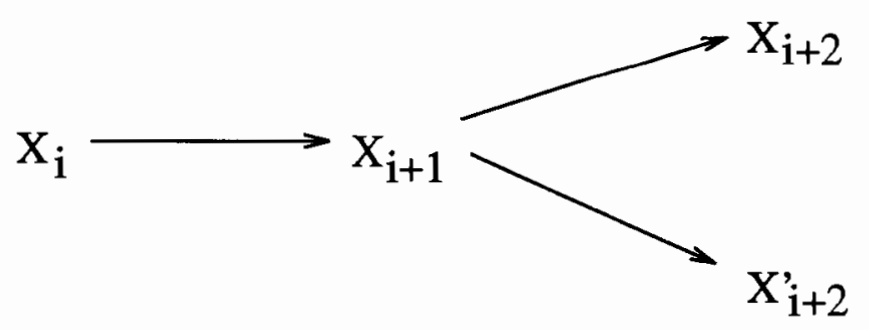

(a)

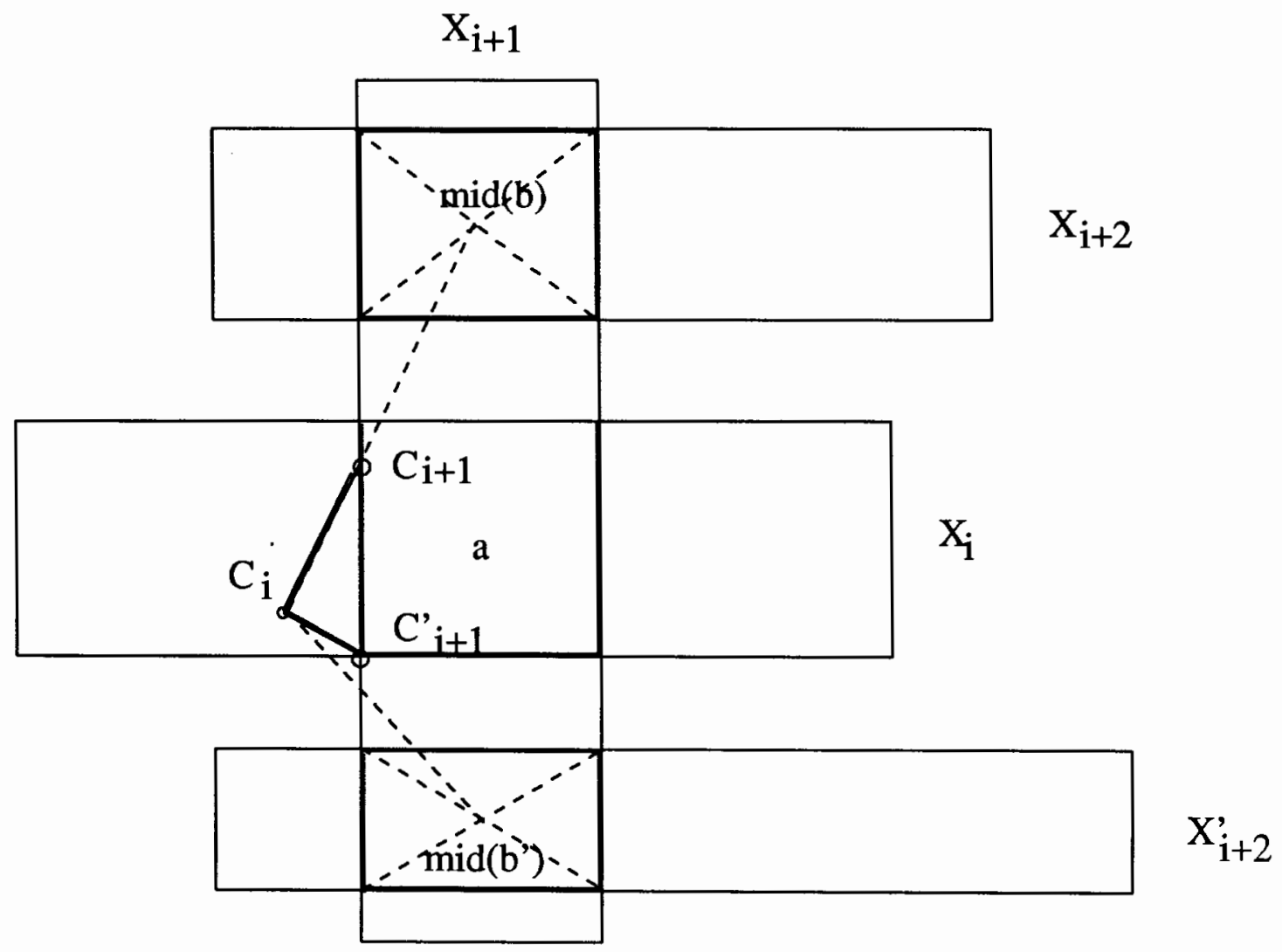

(b)

Figure 2-5. Development of path segment based upon relative position of a future node. (a) Graph path. (b) Corresponding geometric path.

new point $C_{i+1}$ or $C_{i+1}^{\prime}$ is the current path point now and the node path $X_{i} \rightarrow X_{i+1}$ $\rightarrow X_{i+2}$ or $X_{i}>X_{i+1} \rightarrow X_{i+2}^{\prime}$, is added to the current node path. When new point is chosen a cost of path segment $C_{i} \rightarrow C_{i+1}$ is assigned and is added to 
current path cost. A similar procedure will be used to continue path planning until the destination node is reached.

As explained above, the third node $X_{i+2}$ is used to find a new point $C_{i+1}$ in the second node $X_{i+1}$. This is called a one node look ahead. The point path planning and path cost assignments can not begin until the graph node path progress at least to the third node because of a one node look ahead. When the final destination node is reached, the final destination point itself is used in the place of midpoint of a intersection to compute the last two path segments (a path segment from a point in the node right before the destination node to a point $\left(\mathrm{P}_{f}\right)$ on the boundary of final destination node and a path segment from $\mathrm{P}_{f}$ to the destination point) because there is no new node after that final destination node (there is no intersection). Therefore the traversal technique maintains two paths at the same time, one from graph node path and the other from point path.

When the graph node traversal is performed, the next node can be chosen such that an arc exists between the current node and the next node to be progress, and the next node is not on the current node path, and it is not in start nodes, and the arc has not already been considered and deleted during the backtracking procedure. The last case will be explained in next section.

Backtracking. : An exhaustive graph search for the optimal path is performed by using a backtracking procedure. If the following conditions are met, algorithm backtracks by dropping the last node from the current path and continues moving forward along another arc.

1) The path is extended to a destination node, so that the path can not move forward.

2) A new node can not be found. The reason is that nodes connected to the current node may already be on the current path, or has been considered in 
earlier path search and eliminate by some criterion (it will be explained later.).

3) The current cost of the path generated so far exceeds the cost of a source to destination path already established.

Once one path between source to destination is established, a backtrack from a node $\mathrm{B}$ to a node $\mathrm{A}$ that are a part of node path established, implies that all possible ways of going from a node $B$ to one of the destination nodes have been explored. Figure 2-6(a) illustrates some paths from $S$ to $D$. When a path $S \rightarrow A->$ B $\rightarrow$ D is extended, it backtracks to $B$ and a cost associated with an arc B $\rightarrow D$ is assigned to this arc B $->$ D. It is called tcost (traverse cost) which is the tentative minimum cost of reaching a destination node via that arc starting from the emanating node. In this case, tcost is the cost that is a distance from a point in the node B to a destination point. Once a backtrack from D to B is made, then the traversal algorithm continues moving forward along another arc from a node B to a destination node, $\mathrm{B} \rightarrow \mathrm{C} \rightarrow \mathrm{D}$. A backtrack from $\mathrm{D}$ to $\mathrm{C}$ is made and another backtrack from $\mathrm{C}$ to $\mathrm{B}$ if there is no other way from a node $\mathrm{C}$ to $\mathrm{D}$. And a distance from a point in the node $\mathrm{B}$ to the destination point via a point in the node $\mathrm{C}$ is stored in associated with an arc B $->\mathrm{C}$. This traversal and backtracking procedure is progressed till a new node starting from a node B can not be found. Then it backtracks from B to A. This means that all possible ways from a node B to the destination point and consequently all tcosts corresponding to these possible ways have been explored. The minimum cost of a path from $B$ to a destination node can be obtained and consequently the minimum cost ( tcost ) of a path from $\mathrm{A}$ to a destination node via an $\operatorname{arc} \mathrm{A} \rightarrow \mathrm{B}$ which is a distance from a point in the node $\mathrm{A}$ to a point in B plus the minimum tcost from $S$ to $D$.

If the graph arcs had preassigned weights, it would been possible to elim- 


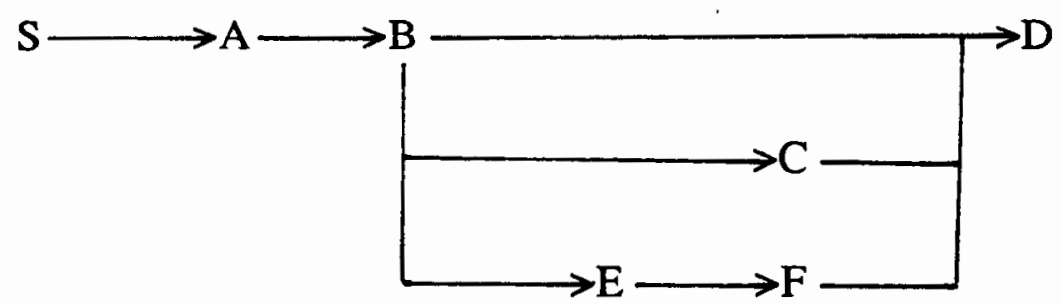

(a)

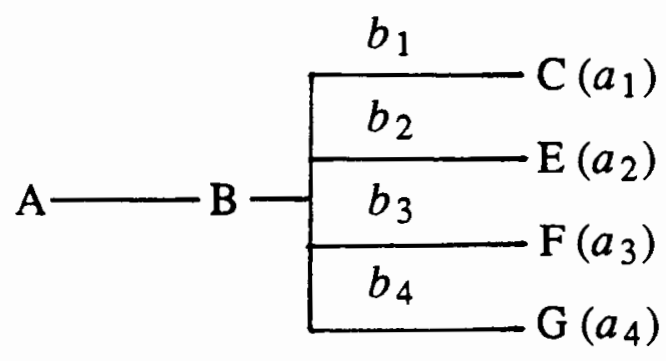

(b)

Figure 2-6. (a) Traversal and backtracking procedure. (b) Deleting links from graph.

inate all but the minimum weight path from all future consideration. However, the costs of these paths depend not only upon a node A but also on the position of the path point in the large area represented by the node A. Since the placement of a point in a node A depends on where the path progress next i.e., the next node, one can no longer assume that one would obtain the same path length from $A$ to the destination in the next visit to $A$. Therefore a parameter $\beta(\beta \geq 1.0)$ is introduced. All those paths whose lengths are within a factor $\beta$ of the minimum path length ( $\beta$ x minimum_tcost) are allowed to survive for future traversals.

Figure 2-6(b) illustrated the process of links being deleted. The backtracking procedure from a node $B$ to a node $A$ is made, which means that one can choose the minimum tcost of reaching the destination. Note that $a_{1}, a_{2}, a_{3}$, and $a_{4}$ are the minimum costs to the destination from C, E, F, and G respectively and 
$b_{1}, b_{2}, b_{3}$, and $b_{4}$ the costs of arcs between B and C, E, F, and G. Assume that $a_{1}+b_{1}$ is the smallest cost compared with other pairs. For all pairs that have a cost that is greater by a factor $\beta$, the corresponding arcs are deleted. This modification is performed while backtracking from node $B$ to node A because that is only time when the exploration of all the alternatives of going from $B$ to the destination is complete.

The considerations outlined above result in the following graph traversal and backtracking algorithm.

Dynamic Cost Allocation Graph Traversal and Backtracking Algorithm.

initialize

bestcost $=\infty$.

currentcost $=0$.

unmark all graph arcs.

Set tcosts for all arcs not emanating from a destination node $=\infty$.

Set tcost all arcs emanating from a destination

$$
\text { node }=0 .
$$

findnewnode $\left[\mathrm{X}_{i+1}\right]$

Choose $X_{i+1}$ such that

an arc exists between $X_{i}$ and $X_{i+1}$, and $X_{i+1}$ is not on current node path, and $X_{i+1}$ is not in $S$, and the arc between $X_{i}$ and $X_{i+1}$ is nat marked.

Set backtrackflag true

if one of the destination nodes is reached, 
or if a new node can not found.

moveforward[to $\left.\mathrm{X}_{i+1}\right]$

Add $\mathrm{X}_{i+1}$ to current node path.

Determine $\mathrm{C}_{i}$ ( as explained in text $)$.

Add $C_{i}$ to current point path.

Add cost segment $\mathrm{C}_{i-1} \rightarrow \mathrm{C}_{i}$ to currentcost.

$i=i+1$.

backtrack[from $\mathrm{X}_{i+1}$ to $\mathrm{X}_{i}$ ]

Unmark all arcs originating from $X_{i+1}$.

Mark ark from $X_{i}$ to $X_{i+1}$.

$\min =$ minmum tcost associated with an arc emanating from $X_{i+1}\left(\right.$ except $\left.X_{i+1} \rightarrow X_{i}\right)$.

if $\min <\infty$, then

delete each $\operatorname{arc} X_{i+1} \rightarrow X_{j}(i \neq j)$

with tcost $>\beta \mathrm{x}$ min

and set tcost of $X_{i} \rightarrow X_{i+1}=\min +$ costsegment

and if currentcost $<$ bestcost, then

bestcost $=$ currentcost

and node path $=$ current node path.

Reduce the currentcost by the cost segment $\mathrm{C}_{i-1} \rightarrow \mathrm{C}_{i}$.

Remove $\mathrm{X}_{i+1}$ from current node path.

Remove $\mathrm{C}_{i}$ from current point path.

$i=i-1$.

path planning (main program)

Determine the source nodes and the destination nodes.

if the source and destination points in same node, then compute straight line path. 
else

initialize.

for every starting node

$i=0$.

backtrackflag $=$ false.

findnewnode $\left[\mathrm{X}_{i+1}\right]$.

while newnode exists or $\mathrm{i}>0$

if backtrackflag = true, then

backtrack $\left[\mathrm{X}_{i+1}\right.$ to $\left.\mathrm{X}_{i}\right]$.

findnewnode $\left[\mathrm{X}_{i+1}\right]$.

end if.

if newnode exists, then

moveforward[to $X_{i+1}$ ].

if currentcost $>$ bestcost, then

backtrackflag $=$ true .

else

findnewnode $\left[\mathrm{X}_{i+1}\right]$

end if.

end if.

end while.

end for.

end if.

\section{ANALYSIS AND MODIFICATIONS}

Performance of the algorithm presented in previous section was evaluate in terms of two characteristics - optimality of the results and speed of computation. 
Near optimality is obtained by taking into consideration all possible nodes and by conducting a complete search of the graph obtained. These very same considerations unfortunately also imply poor execution speed. Since both optimality and speed may not be realized at the same time, the parameter $\beta$ is used to systematically trade off optimality for execution speed.

Recall that $\beta$ determines which graph arcs are deleted while backtracking. If $\beta$ is 1 , the backtracking algorithm eliminates all but the minimum weight arc going out from backtracked node at every backtrack. However, the costs of these paths depend not only upon the node but also on the position of path point in that node. This assumption leads that arcs that might be a part of optimal path in the future path search, could be deleted, and the result obtained with $\beta=1$ is suboptimal path due to a highly restricted search. Another problem is that the path search is dependent on the structure of the node graph, the order of nodes to access, which means that certain arcs could be deleted in one order of access but not in another order of access. When two identical models are used to plan path, one could have different results with two different structures of the graph. This is because a highly restricted search is performed. Figure 2-7 shows the problems caused by highly restricted search. This figure illustrates two paths: The one starts from $(50,95)$ to $(20,5)$ and the other from $(20,5)$ to $(50,95)$. The results established is different paths because of restricted search. (After modifications, two different paths can have the same route which is the point path of $(20,5)<->(30$, $10) \leftrightarrow(50,95)$.) As $\beta$ is larger, the number of arcs removed is decreased. Finally, with very large value of $\beta$, no arcs are deleted and the search for the optimal path proceeds through all possible travel pattern. In other word, as the value of $\beta$ increases, the two problems mentioned above can be solved and paths tend to get closer to optimal but the computational time required increase because 
of an exhaustive search.

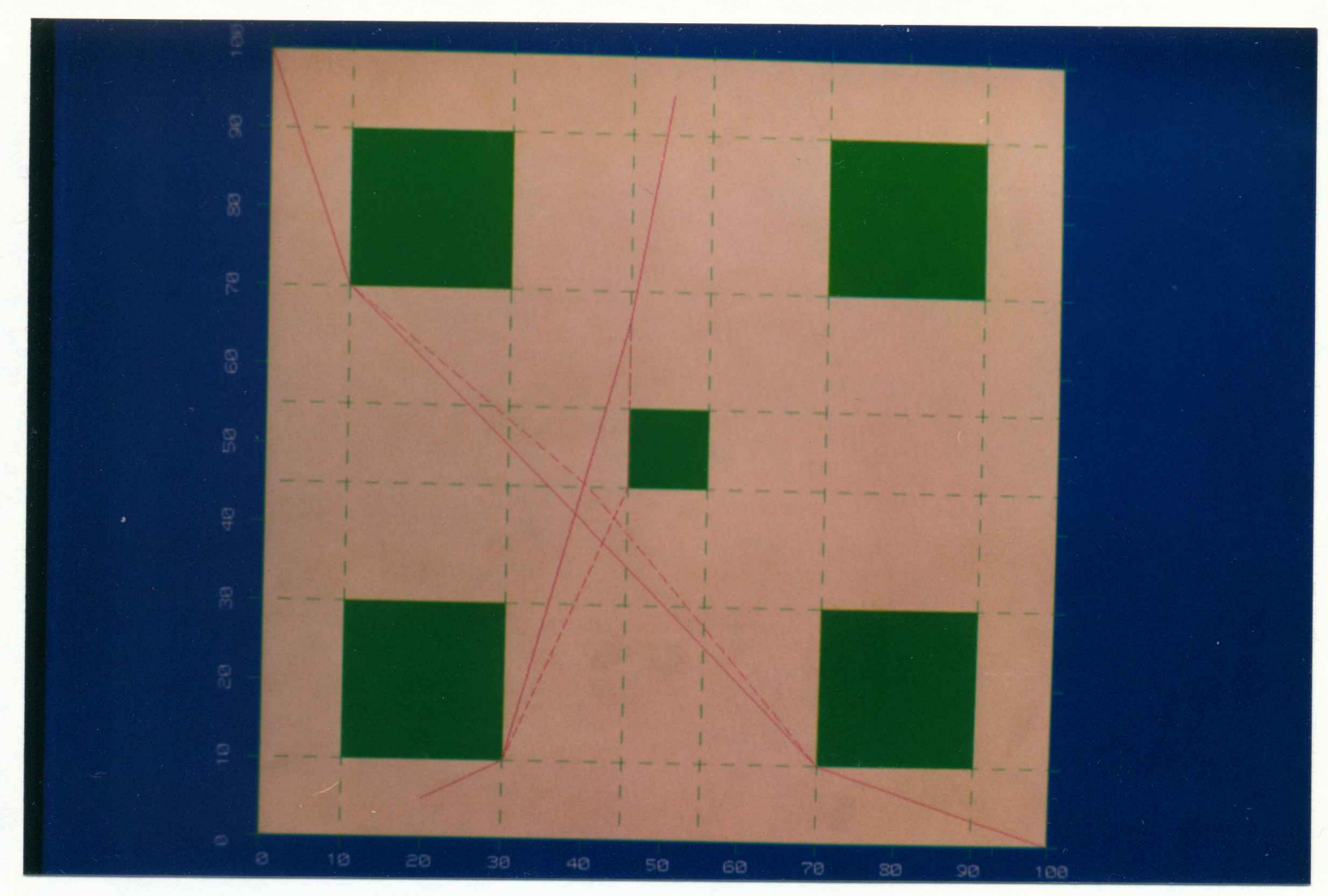

Figure 2-7. Sample layout resulted from a highly restricted search.

In many case of path planning, the path obtained make wide turns at the near corners of obstacles regardless of the value of $\beta$ because of the traversal algorithm. When a one node look ahead is used, the next path point chosen falls on the boundary of intersection a node $X_{i}$ and the next node $X_{i+1}$, which is dependent on where the path will progress next. If the placement of this point within the intersection is on the side of the boundary, not on the corner of boundary, then this wide turn happens at the near corners of obstacles.

In the case of $\beta=1$, taking advantage of the smallest computational time and the suboptimal path, the path planning algorithm can be modified by using 
obstacle areas with small increase of execution time (approximately 0.1 to 0.2 $\mathrm{sec})$. Once suboptimal path from the source to the destination is established with $\beta=1$, the modification starts using each point in the point path already established.

modification 1 : Assuming that the point traversal is $\mathrm{C}_{i} \rightarrow \mathrm{C}_{i+1} \rightarrow \mathrm{C}_{i+2}->$ $\mathrm{C}_{i+3}$---- and start from the point $\mathrm{C}_{i}$, points $\mathrm{C}_{i}$ and $\mathrm{C}_{i+2}$ are joined by a straight line. If the line does not intersects any obstacle areas, then the point $\mathrm{C}_{i+1}$ is moved to a point on the line connecting $\mathrm{C}_{i}$ with $\mathrm{C}_{i+2}$, which is closest to $C_{i+1}$ (if $C_{i}=C_{i+1}$, then the point $C_{i+1}$ is removed from the point path.). And the point $C_{i}$ is connected with $C_{i+3}$ by a straight line and the point $\mathrm{C}_{i+2}$ is moved on the line if the line connecting $\mathrm{C}_{i}$ with $\mathrm{C}_{i+3}$ is in the free space. The same procedure is proceeded till the line intersects any obstacles areas. Assume that the modification is extend to a point $C_{j}$ and the line $\left(l_{1}\right)$ connecting $C_{i}$ with $C_{j}$ intersects obstacle areas. At this point, it is better to check if the line $\left(l_{2}\right)$ connecting $C_{i}$ with $C_{j+1}$ intersects any obstacle areas since $l_{2}$ is often collision free line segment even though $l_{1}$ is not. In figure $2-7$, the line segment, $(50,95) \rightarrow(30,10)$, is in free space but the segment, $(50,95) \rightarrow(45,45)$, is not. If $l_{2}$ is collision free, then keep moving forward. If not, then start the same procedure from the previous point of that point connected with the point $C_{i}$. Points $C_{i}$ and $C_{i+2}$, for example, are joined by a straight line, and the line intersects obstacles. Then continue finding free space from the point $C_{i+1}$. This modification is extended from starting point to destination point. The path d-o in figure 2-9 shows this modification.

modification 2 : When two adjacent points $C_{i}$ and $C_{i+1}$ are in a row vertically or horizontally, and the point $C_{i}$ is not on the corner of obstacle, and the line lies on the side of boundary of obstacle area, move the point $C_{i}$ to 


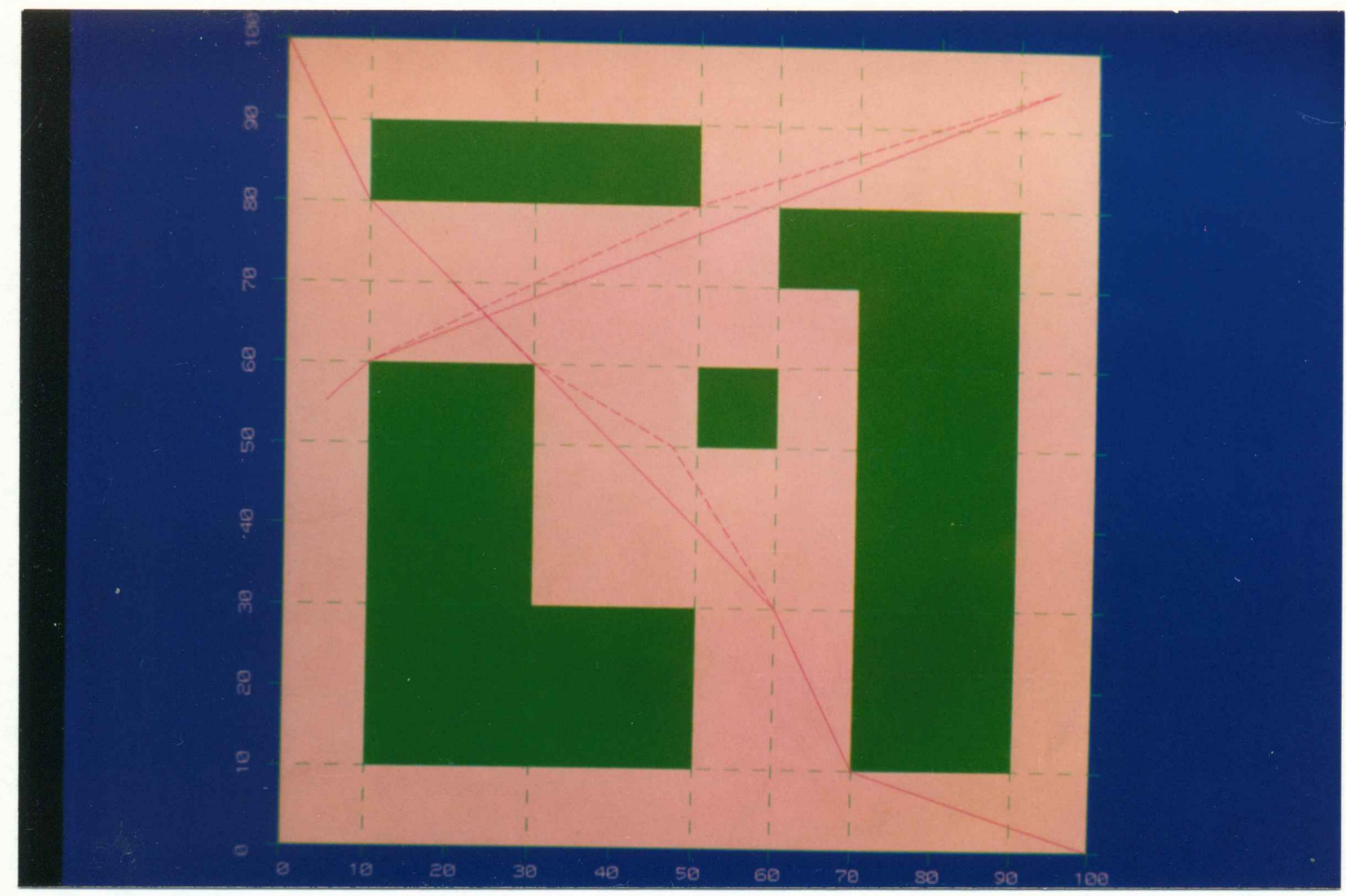
Figure 2-8. Sample layout resulted from the modification 1 which is ap-
plied once.

the corner of the obstacle that is closest to the line if the straight line connecting the corner with the point $\mathrm{C}_{i-1}$, does not intersect any obstacle areas. The path b-m in figure 2-9 illustrates this case.

modification 3 : If a point $\mathrm{C}_{i}$ is adjacent to a corner of the obstacle vertically or horizontally, then another modification is applied. Assume that the path segments that is sum of lines connecting $C_{i-1}$ with $C_{i}$ and $C_{i}$ with $\mathrm{C}_{i+1}$ is $\mathrm{L}_{1}$. The point $\mathrm{C}_{i}$ can be moved to the corner of obstacle if two straight lines ( $\mathrm{L}_{2}$ is the sum of two lines) that are connecting the point $\mathrm{C}_{i-1}$ with the corner and the corner with $\mathrm{C}_{i+1}$, do not intersect obstacle areas 


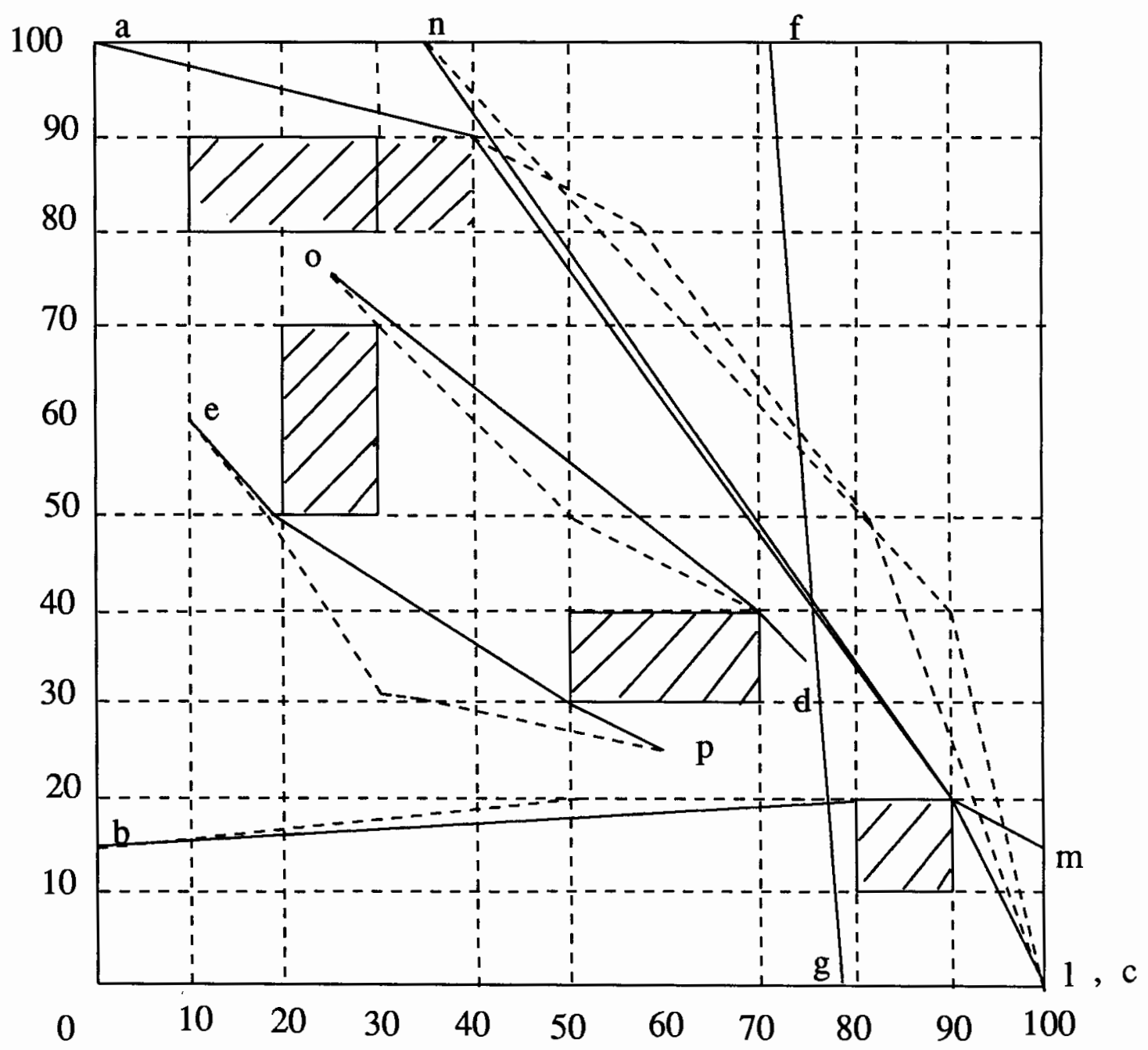

Figure 2-9. Sample layout with 4 obstacles and sample paths obtained by procedure of this paper for $\beta=1$ (dotted lines ) and modified ( solid line ).

and if $\mathrm{L}_{2}$ is smaller than $\mathrm{L}_{1}$. The path $\mathrm{c}-\mathrm{n}$ in figure 2-9 shows this modification.

The modification 1 and 2 can fix most of problems suboptimal path with $\beta=1$ has, caused by a highly restricted search and the structure of the node graph. And the modification 3 improves the wide turn around corners of obstacles caused by the traversal procedure. The path a-1 in figure $2-9$ is the combined case, 


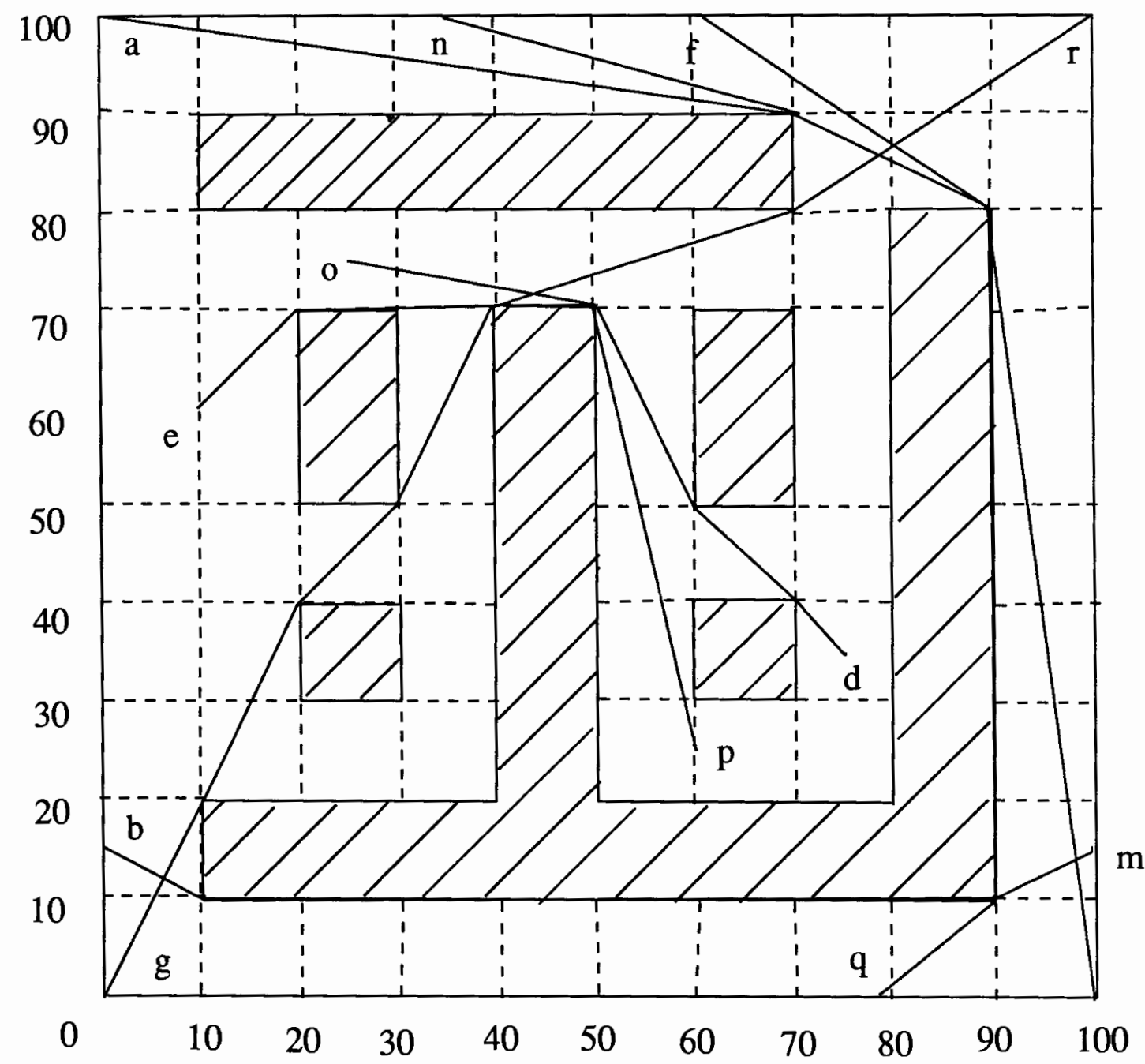

Figure 2-10. Sample cluttered layout and sample paths obtained by procedure of this paper for modified method with $\beta=1$.

modification 1 and modification 3. The modification 1 may be repeated twice or three times to increase optimality as this modification is extended from the very next point $\left(\mathrm{C}_{s+1}\right)$ of starting point to destination point or from $\mathrm{C}_{s+2}$ to destination point, respectively since there might be another $C_{i} \rightarrow C_{j}\left(C_{i}\right.$ and $C_{j}$ are joined by a straight line.) after the modification 1 is extended first. The solid line path $(0$, $100)$ to $(100,0)$ in figure $2-8$, for example, is the path after the modification 1 is 
applied first. If modification 1 is applied from the point $(10,80)$ to $(100,0)$, then a straight line segment from $(10,80)$ to $(70,10)$ is obtained, which is more optimal. And the path e-p in figure 2-9 shows that the modification 1 is applied twice. Using these modifications, near optimality is obtained without increasing computational time. Figure 2-9 shows the layout and the paths produced between six pairs of the source and destination points for $\beta=1$ and modified paths. Four obstacles are partitioned into $9 \times 9$ grid by distint edges of objects. Table 2-I compares the costs of paths obtained for $\beta=1$ and 2 with those of the modified method. It also lists the amount of time required by the algorithm (implemented in $\mathrm{C}$ running under Unix on a VAX 11/780) to complete each path. As can be seen from the table, modified paths are very close to optimal and the computational time required is relatively fast.

\section{TABLE 2-I}

COMPARISON OF PATH LENGTHS AND COMPUTATIONAL TIME OBTAINED BY $\beta=1$ AND $\beta=2$ WITH THOSE OBTAINED BY MODIFIED METHOD FOR LAYOUT OF FIG.2-9.

\begin{tabular}{|c|rrr|ccc|}
\hline $\begin{array}{c}\text { Sample } \\
\text { Path }\end{array}$ & \multicolumn{3}{|c|}{ Path Length } & \multicolumn{3}{c|}{$\begin{array}{c}\text { Computation Time } \\
\text { (sec) }\end{array}$} \\
\hline \hline $\mathrm{a}-1$ & 152.8 & 148.3 & 149.6 & 2.0 & 16.6 & 2.1 \\
$\mathrm{~b}-\mathrm{m}$ & 101.4 & 101.4 & 101.3 & 2.0 & 6.3 & 2.0 \\
$\mathrm{c}-\mathrm{n}$ & 122.6 & 122.6 & 119.4 & 1.4 & 4.3 & 1.5 \\
$\mathrm{~d}-\mathrm{o}$ & 64.8 & 64.1 & 64.1 & 1.9 & 6.8 & 2.0 \\
$\mathrm{e}-\mathrm{p}$ & 65.7 & 61.4 & 61.6 & 2.1 & 4.8 & 2.1 \\
$\mathrm{f}-\mathrm{q}$ & 100.3 & 100.3 & 100.3 & 0.2 & 0.2 & 0.2 \\
\hline
\end{tabular}

Time required to set up graph and database was $0.6 \mathrm{sec}$. 
Figure 2-10 shows the same paths in a cluttered environment obtained by adding more obstacles to the layout of figure 2-9. However, this addition has been done without changing the grid by aligning the new objects with objects already present. Optimality of the resultant paths and the computational time for this layout are presented in Table 2-II. It can be seen that the parameter $\beta$ has the same effect in a cluttered environment. A comparison of Table 2-II with Table 2-I shows that the computational times required in a cluttered environment are substantially lower than in a simple environment. The graph corresponding to layout of figure 2-9 has 16 nodes and 140 directed arcs, whereas that of figure 2-10 has 14 nodes and 48 directed arcs. Four different environment with sample paths for $\beta=1$ (dotted line) and modified (solid line) are illustrated in figure 2-11.

TABLE 2-II

PATH LENGTH AND COMPUTATIONAL TIME OBTAINED BY USING MODIFIED METHOD FOR LAYOUT OF FIG.2-10.

\begin{tabular}{|ccc|}
\hline $\begin{array}{c}\text { Sample } \\
\text { Path }\end{array}$ & $\begin{array}{c}\text { Modified } \\
\text { Path Length }\end{array}$ & $\begin{array}{c}\text { Computational } \\
\text { Time(sec) }\end{array}$ \\
\hline \hline $\mathrm{a}-1$ & 173.9 & 1.0 \\
$\mathrm{~b}-\mathrm{m}$ & 102.4 & 1.1 \\
$\mathrm{c}-\mathrm{n}$ & 139.4 & 0.6 \\
$\mathrm{~d}-\mathrm{o}$ & 69.1 & 0.5 \\
$\mathrm{e}-\mathrm{p}$ & 90.2 & 0.5 \\
$\mathrm{f}-\mathrm{q}$ & 114.3 & 0.8 \\
$\mathrm{~g}-\mathrm{r}$ & 148.9 & 0.6 \\
\hline
\end{tabular}

Time required to set up graph and database was $0.8 \mathrm{sec}$.

Time Complexity : In order to understand the relationship of the computa- 
tional time to the problem parameters, nine different environments with five nontrivial paths in each were simulated. The results shown in Table 2-III indicate that the computational time depends on the number of nodes and the number of arcs. It is also clear that the time is more strongly dependent upon the number of node than on the number of arcs.

TABLE 2-III

RELATIONSHIP BETWEEN GRAPH PARAMETERS AND PATH COMPUTATIONTAL TIME ( AVERAGE OVER FIVE NONTRIVIAL PATHS ).

\begin{tabular}{|ccccc|}
$\begin{array}{c}\text { Number of } \\
\text { Objects }\end{array}$ & $\begin{array}{c}\text { Grid } \\
\text { Size }\end{array}$ & $\begin{array}{c}\text { Number of } \\
\text { Graph Nodes }\end{array}$ & $\begin{array}{c}\text { Number of } \\
\text { Graph Arcs }\end{array}$ & $\begin{array}{c}\text { Average } \\
\text { Computation Time(s) }\end{array}$ \\
\hline \hline 3 & $7 \times 7$ & 13 & 96 & 1.02 \\
4 & $9 \times 9$ & 16 & 140 & 1.94 \\
5 & $7 \times 7$ & 12 & 72 & 0.74 \\
6 & $8 \times 8$ & 12 & 44 & 0.44 \\
7 & $7 \times 7$ & 11 & 36 & 0.34 \\
8 & $10 \times 10$ & 19 & 126 & 2.3 \\
8 & $10 \times 10$ & 14 & 48 & 0.80 \\
9 & $9 \times 9$ & 17 & 76 & 0.94 \\
9 & $11 \times 11$ & 13 & 44 & 0.58 \\
\hline
\end{tabular}

The exact relationship between the computational time and the number of nodes is very difficult to derive. Instead, an upper bound for the number of nodes in the node graph has been derived as follows. Assume that there are n nonoverlapping objects with no edges aligned. The layout is then partitioned by the edges of the obstacles into $(2 n+1) \times(2 n+1)$ grid. Note that each prime convex area is 
bounded at least on one side of an obstacle. There is only one prime convex area that is bounded by both the west side of $\mathrm{E}$ and the east side of $\mathrm{W}$ for any pair of obstacles, $\mathrm{E}$ and $\mathrm{W}$ (with $\mathrm{E}$ on the east of $\mathrm{W}$ ). This is because there is only one prime convex area that is not fully incorporated in any other single prime convex area (the definition of prime convex area), enclosed by the same pair of objects, $\mathrm{E}$ and $\mathrm{W}$. To estimate the number of prime convex areas bounded either on the east or the west of an obstacle, the number of ways in which $\mathbf{n}$ objects and the east and west boundaries of the layout can be paired should be considered. Therefore the number of ways is the number of combinations of $(n+2)$ elements taken 2 at a time, which is

$$
(n+2)(n+1) / 2
$$

However, the area enclosed by the pair made up of the east-west boundaries of the layout need not be considered since only areas which are enclosed by either east or west of layout and either east or west of obstacles are counted. Thus there are

$$
\begin{gathered}
(\mathrm{n}+2)(\mathrm{n}+1) / 2-1 \\
=\mathrm{n}(\mathrm{n}+3) / 2
\end{gathered}
$$

such prime convex areas. The same number of prime convex areas can be accounted for by a north-south argument. Thus the number of prime convex areas is bounded above by

$$
\mathrm{n}(\mathrm{n}+3)
$$

which is the time complexity of

$$
\mathrm{O}\left(\mathrm{n}^{2}\right)
$$


Note that this is a loose upper bound since many pairs of faces counted in the above argument may not indeed give rise to a free convex area and some areas may be counted twice, once in the east-west argument and once in the north-south argument. In most of practical cases, the set up is cluttered with aligned objects. This result in the reduction of many of prime convex areas and arcs which represent the intersections of prime convex areas. Thus a faster search through the graph is resulted and consequently the speed is improved for the procedure. 

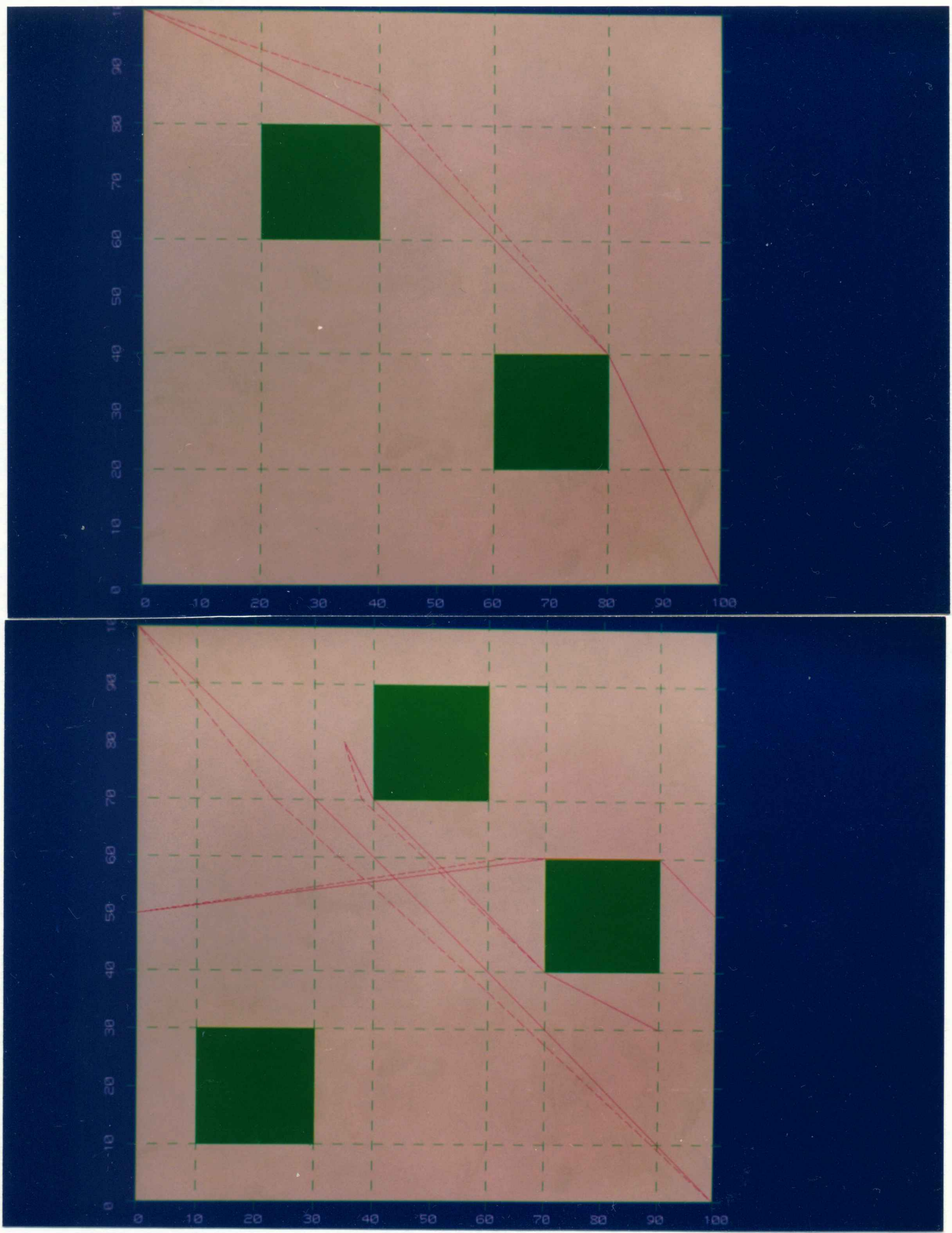

Figure 2-11. Sample layouts and sample paths obtained by procedure of this paper for $\beta=1$ (dotted line) and modified (solid line). 


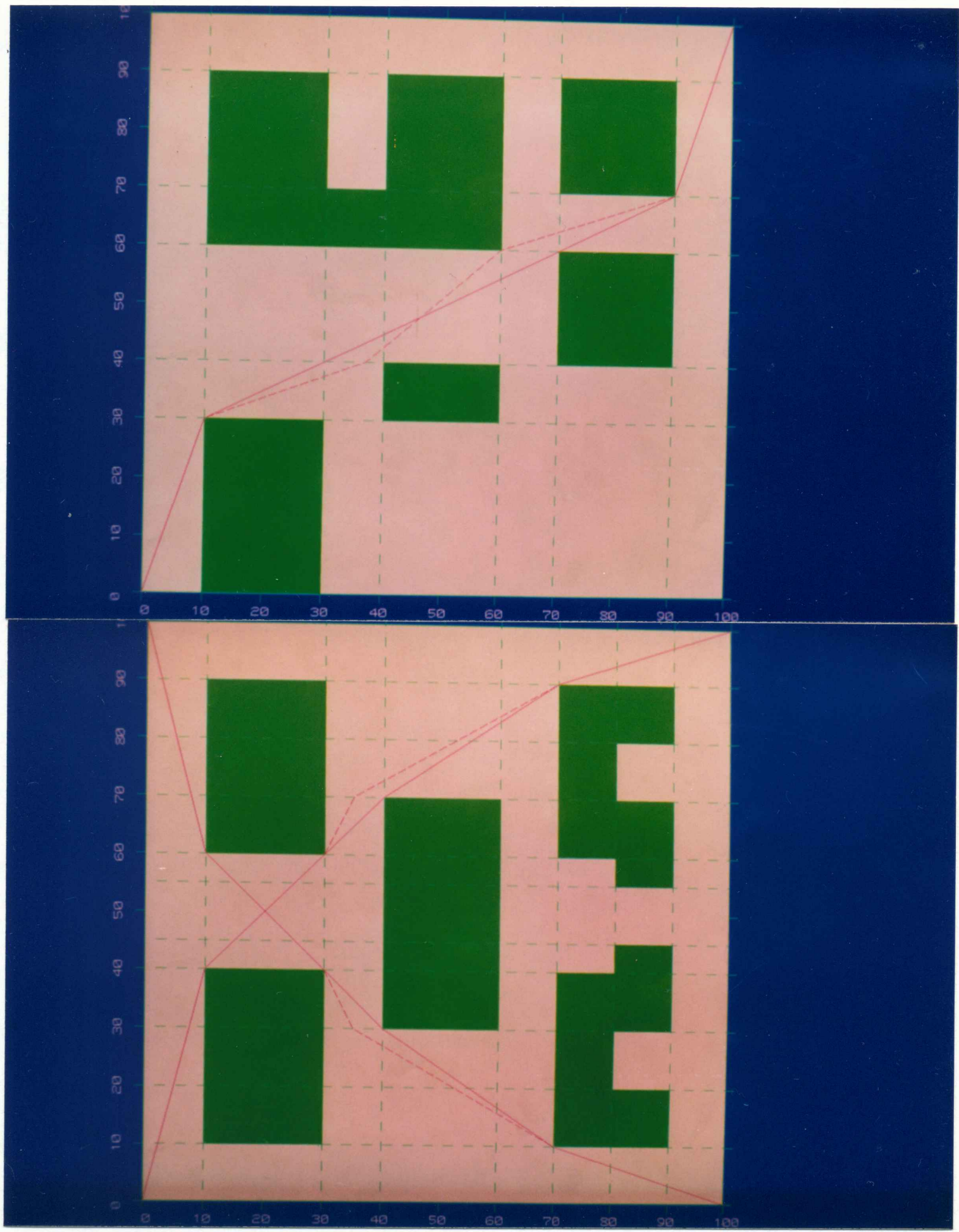

Figure 2-11. (continued) Sample layouts and sample paths. 


\section{CHAPTER III}

\section{THREE DIMENSIONAL \\ PATH PLANNING}

In proposed three-dimensional path planning approach, the recognition and reconstruction of three-dimensional models for the obstacles in the workspace is not desirable. Rather it is proposed to model the environment by a set of three orthogonal two-dimensional projections of the three-dimensional workspace. Three-dimensional collision checking is decomposed into the problem of twodimensional collision checking in the three orthogonal two-dimensional projections.

\section{THREE ORTHOGONAL SPACE PROJECTIONS}

Similar to the concept of partitioning the two-dimensional layout in twodimensional path planning, the three-dimensional workspace can be partitioned by the edges of obstacles into box-shape free spaces in three-dimensional path planning. A prime free space is a space that is free of obstacles, has the property that any two points in that three-dimensional free space can be joined by a straight line that lies entirely within that area, and is not fully incorporated in any other single prime free space. This prime free space is called the three-dimensional node (to distinguish it from two-dimensional node used in two-dimensional path planning) in three-dimensional path planning.

In a direct approach to three-dimensional path planning based on visual input obtained from the environment, the three-dimensional recognitions or 
reconstructions of all the obstacles in the workspace may not always be possible with a finite number of views because of occluding effects and insufficient information Even though they are possible, the recognition and reconstruction processes are computationally expensive. Rather, by knowing where there are obstacles and where there is free space in three-dimensional workspace using three orthogonal two-dimensional subspaces, a set of two-dimensional projections is better to be used to represent the three-dimensional workspace. These three orthogonal subspaces projected orthogonally from top and two sides of threedimensional workspace are called xy-plane, $x z$-plane, and yz-plane, respectively.

A box-shape hexahedron is composed into six faces of rectangles. Knowing the two out of six faces of rectangles is enough to determine the shape and size of box. Normally, each box-shape three-dimensional node should correspond to unique combination of two rectangle-shape two-dimensional nodes in two out of the three orthogonal subspaces, which are partitioning two of six faces of threedimensional free spaces. In other word, it can be also said that a three-dimensional node can be decomposed into a set of two two-dimensional nodes in the three subspace. However, because of the occluding effects, a face corresponding to a boxshape free space may be absent in one or more of the two subspaces and it lies over the area occupied by obstacles. This results in some three-dimensional nodes not having all two faces in the two subspaces (resulted from its decomposition) present in the three orthogonal subspaces. That is, the projection of a threedimensional node may be occluded and therefore, not present as two-dimensional nodes in one or more of the orthogonal subspaces.

As explained above, the prime convex areas in three two-dimensional projections represent the three-dimensional nodes even though they do not provide all the three-dimensional nodes because of occluding effects. A three-dimensional 
path planning algorithm is developed using all informations from three orthogonal subspaces. These two-dimensional layouts containing the prime convex areas and the obstacle areas are obtained by applying Quine-McClusky (10) method to each of three two-dimensional subspaces. And the node graphs for each of three twodimensional subspaces are set up such that two two-dimensional nodes in corresponding two-dimensional subspace are joined by an arc if the areas they represent intersect.

\section{PATH PLANNING}

A prime convex area in each of subspaces corresponds to threedimensional node, i.e., a two-dimensional node is a collision free space when it is extended to three-dimensional space. Therefore, a collision free path established in one two-dimensional projection can be extended to a three-dimensional collision free path by using informations from the other two two-dimensional subspaces.

The basic strategy of three-dimensional robot path planning involves obtaining at most three two-dimensional paths from each of three two-dimensional subspaces, extending each of those paths to a three-dimensional path, choosing the minimum cost of three-dimensional path, and modifying the three-dimensional path established by knowing where there are collision free space in threedimensional workspace. This collision checking modification is performed in three orthogonal projections.

Obtaining a Three-Dimensioanl Path : Three paths is established in each of three subspaces from the source to the destination that are corresponding to the projections of the three-dimensional source and destination points, where the traversal and backtracking procedure is applied to each path planning and each 
two-dimensional path is modified by using the method introduced in chapter II. Normally a point in three-dimensional workspace should correspond to a point in each of the orthogonal subspaces. However, because of the occluding effects, a point corresponding to a three-dimensional point may lie inside a obstacle area in one or more of the orthogonal subspaces. In two-dimensional path planning, both the source and destination points should fall inside two- dimensional nodes. But one or both of the source and destination points may lie inside obstacle areas in one or more of the subspaces. In the worst case, one or both of the source and destination points are embedded in all the three subspaces, and no two-dimensional path can be found in any of subspaces. Here, a assumption is made to avoid the problem mentioned above: Both of the source and destination points can not lie inside obstacle areas in $x y$-plane projected from the top of the three-dimensional workspace, which means that the objects moved do not start from above the obstacles or terminate above obstacles. Therefore there exists at least one twodimensional path in three orthogonal subspaces. Once at most three twodimensional paths are established, three-dimensional paths can be constructed by using the other two two-dimensional subspaces. For example, once a twodimensional path is established in the xy-plane, the source and destination points are connected by a straight line in xz-plane and yz-plane. Then the longer straight line is chosen as a decomposed two-dimensional path. Using the two decomposed paths, the path in xy-plane and the straight line in xz-plane or yz-plane, a threedimensional path is constructed. The same procedure is applied if there exist the source and destination points inside free areas in xz-plane or yz-plane. The final three-dimensional path chosen is the path that has minimum cost of path among at most three three-dimensional paths, where each of those paths is based on two decomposed two-dimensional paths, a two-dimensional path in one twodimensional subspace and a straight line path in one of the other two two- 
dimensional subspaces. In addition, if the source and the destination points belong to the same two-dimensional node in any two-dimensional subspace, the threedimensional path is a straight line since two straight lines, one from twodimensional path planning in one two-dimensional subspace and a straight line in other two-dimensional subspace, construct a straight line three-dimensional path.

Modifications : Once a three-dimensional path from the source to the destination is established, the modifications start by using each point in the threedimensional point path already obtained. This method is very similar to the modification 1 explained in chapter II, but extend the same idea to the three orthogonal subspaces. There is a important Lamma to modify this threedimensional path.

Lamma : If the projection of some three-dimensional path for the threedimensional moving object is collision free in one or more of the three orthogonal projections, then the three-dimensional moving object is collision free along the three-dimensional path in the three-dimensional space.

Assuming that the three-dimensional point path traversal is $\mathrm{C}_{i} \rightarrow \mathrm{C}_{i+1} \rightarrow$ $\mathrm{C}_{i+2} \rightarrow \mathrm{C}_{i+3}-\cdots$, and the modification starts from the point $\mathrm{C}_{i}$, the projected points, $\mathrm{Cp}_{i}$ and $\mathrm{Cp}_{i+2}$ of the three-dimensional points $\mathrm{C}_{i}$ and $\mathrm{C}_{i+2}$ are joined by a straight line in each of three orthogonal subspaces. If the line does not intersect any obstacle areas in one or more of three orthogonal projections, then this threedimensional path segment is collision free, the three-dimensional point $C_{i+1}$ is marked, and the procedure continues moving forward to $\mathrm{C}_{i+3}$. The projected point $\mathrm{Cp}_{i}$ is connected with $\mathrm{Cp}_{i+3}$ by a straight line in each of three subspaces and the point $\mathrm{C}_{i+2}$ is marked if the line is collision free in one or more of three orthogonal subspaces. The same procedure is proceeded till there is no collision free line 
segment in all the three orthogonal subspaces. The modification is proceeded to the point $\mathrm{C}_{j}$ and the straight line connecting $\mathrm{Cp}_{i}$ with $\mathrm{Cp}_{j}$ intersects obstacle areas in all the three subspaces. In two-dimensional case, the modification procedure start finding collision free path segment from the point $\mathrm{C}_{j-1}$. On the other hand, in three-dimensional case, the straight line connecting two points $\mathrm{Cp}_{i}$ and $\mathrm{Cp}_{j}$ that are not adjacent, could be a collision free path segment, even though the line intersects the obstacle areas in all the three orthogonal subspaces. This is because each partition $\left(\mathrm{C}_{i} \rightarrow \mathrm{C}_{i+1}, \mathrm{C}_{i+1} \rightarrow \mathrm{C}_{i+2},----\right.$, and $\left.\mathrm{C}_{j-1}->\mathrm{C}_{j}\right)$ of the line could be collision free in one or more of three subspaces when the line connecting two projected points $\mathrm{Cp}_{i}$ and $\mathrm{Cp}_{j}$, is divided into several partitions by the points ( $\mathrm{x}$ points of path points in $x y$-plane, $x$ points in $x z$-plane, and y points in yz-plane ) between $\mathrm{Cp}_{i}$ and $\mathrm{Cp}_{j}$ in three orthogonal subspaces.

The collision checking procedure mentioned above is applied to between two points $C_{i}$ and $C_{j}$. If there exists collision free path, then mark the threedimensional point $C_{j-1}$ and move forward to $C_{j+1}$. And if not, then the marked points between $C_{i}$ and $C_{j}$ are removed from the points path and continue collision checking from a three-dimensional point $\mathrm{C}_{j-1}$. This collision checking modification is extended from the starting point to the destination point.

Figure 3-1 shows that the difference between an unmodified threedimensional path and a modified one where the dotted line is the unmodified path and the solid line is the modified one.

\section{ANALYSIS AND SIMULATION OF THE ALGORITHM}

With the above proposed method for three-dimensional path planning, a set of two-dimensional orthogonal subspaces have been used to represent the threedimensional workspace. The decomposed paths have been established from the 
three subspaces and the process of three-dimensional collision checking modification has been decomposed into the simpler process of two-dimensional collision checking. This process is simpler than forming three-dimensional geometric models of obstacles from two-dimensional images of the workspace and try to determine if a three-dimensional path intersects any of the three-dimensional obstacles.

The three-dimensional path planning algorithm was implemented in $\mathrm{C}$ running under Unix on a VAX 11/780. The three-dimensional algorithm was successful in finding three-dimensional path as illustrated in figure 3-2, 3-3, and 3-4 which correspond to the three orthogonal subspaces of three-dimensional workspace and reconstruction of three-dimensional obstacles and the threedimensional path found. In most cases of three-dimensional path planning, the three-dimensional path established is a near optimal path. However, in a very cluttered environment, the occluding effect may be so severe that much of the information is lost in one or more of the orthogonal subspaces and a path could not be found even one exists in reality. Figure 3-5 shows this case of nonoptimal path. In such cases, cameras may be moved at a viewing angle (other than the orthogonal angles) favorable to obtain additional information about the environment, or some additional cameras with three orthogonal cameras already present may be installed at various different viewing angles. The question is then at what angles it is favorable to obtain additional information of free space and how to transform projections taken at angles different from the orthogonal angles (or at additional angles from additional cameras) into orthogonal subspaces representation. This remains as future works.

Time Complexity : As the dependence of graph parameters and path computational time in the two-dimensional time derivation, the three-dimensional time 
complexity is strongly dependent upon the number of three-dimensional nodes. However, the relationship between the number of node and the computational time should be calculated in terms of two-dimensional nodes because a set of three orthogonal two-dimensional projections is used to represent the three-dimensional workspace. Upper bounds for the number of two-dimensional nodes in each graph of three projections can be derived as explained in chapter II. Here, the same assumption has to be made to get upper bounds. There are $\mathrm{n}$ nonoverlapping objects with no edges aligned in three-dimensional workspace. Then each subspace is partitioned by the edges of the projected objects because no objects are overlapped and aligned and each subspace has $\mathbf{n}$ projected obstacles. Therefore the number of two-dimensional nodes is bounded above by $n(n+3)$ in each of three subspaces and the total number of two-dimensional nodes is $3 n(n+3)$. However, in the most workspaces, the objects usually stand on the ground. This means that there are no ways in which objects and south boundary of subspace can be paired in xz-plane and yz-plane. Therefore the number of ways is the number of combinations of $(n+1)$ elements taken two at a time in a north-south argument in xzplane or yz-plane. And considering the area enclosed by the pair made up of north-south boundaries of a subspace the number of two-dimensional nodes for $\mathrm{xz}$-plane or yz-plane is

$$
\begin{aligned}
& \{(n+2)(n+1) / 2-1\}+\{(n+1) n / 2-1\} \\
& =n(n+3) / 2+\{n(n+1)-2\} / 2 \\
& =n^{2}+2 n-1
\end{aligned}
$$

The total number of two-dimensional node for three-dimensional workspaces is the sum of the two-dimensional nodes obtained from $x y$-plane, xz-plane, and yzplane, which is 


$$
\begin{aligned}
& \{n(n+3)\}+\left\{n^{2}+2 n-1\right\}+\left\{n^{2}+2 n-1\right\} \\
& =3 n^{2}+2 n-2
\end{aligned}
$$

Therefore the time complexity for three-dimensional is

$$
O\left(3 n^{2}\right)
$$

This is also a loose upper bound because of the reason explained in chapter II. 

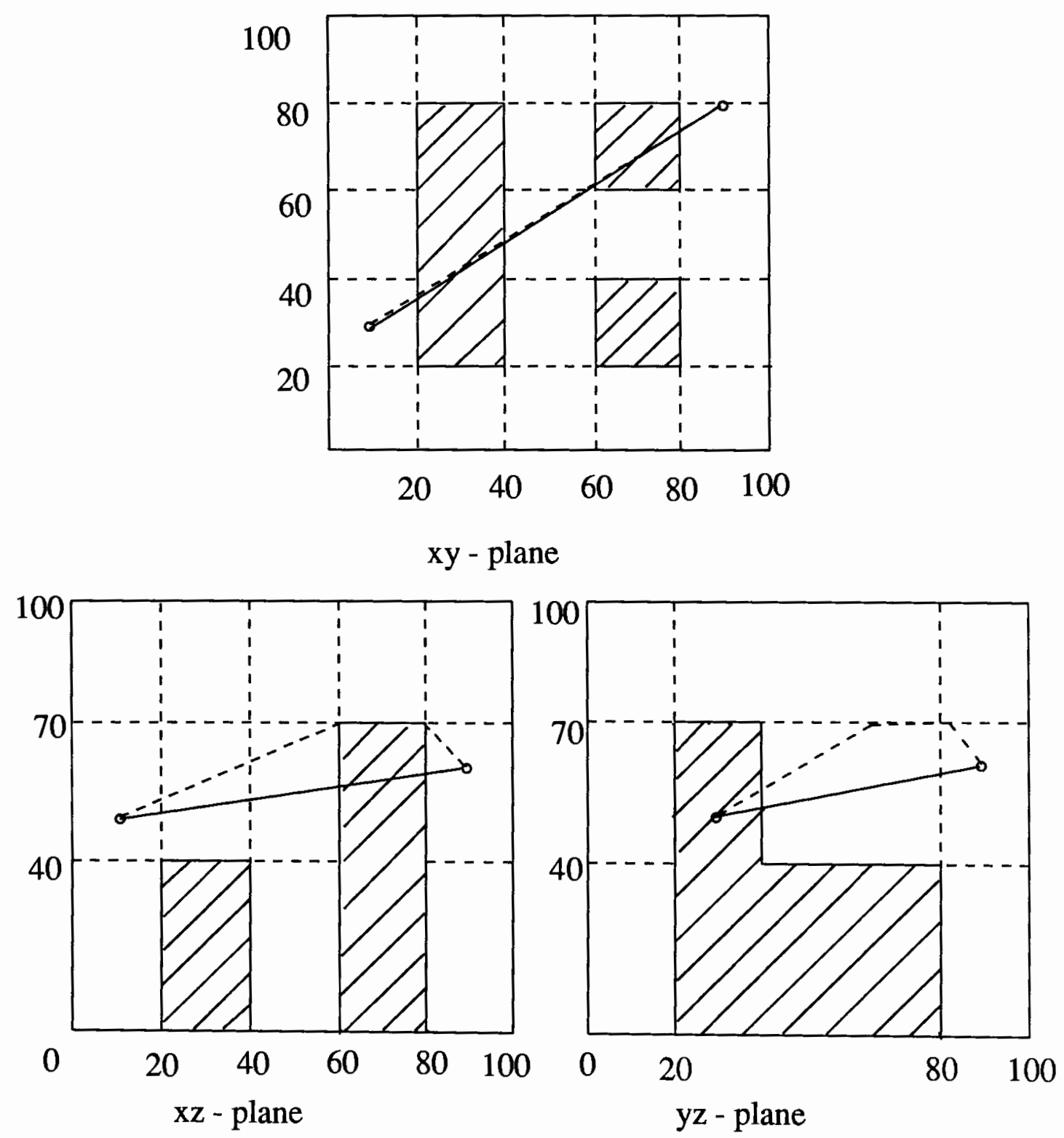

Figure 3-1. The difference between an unmodified path (dotted line) and a modified one ( solid line). 


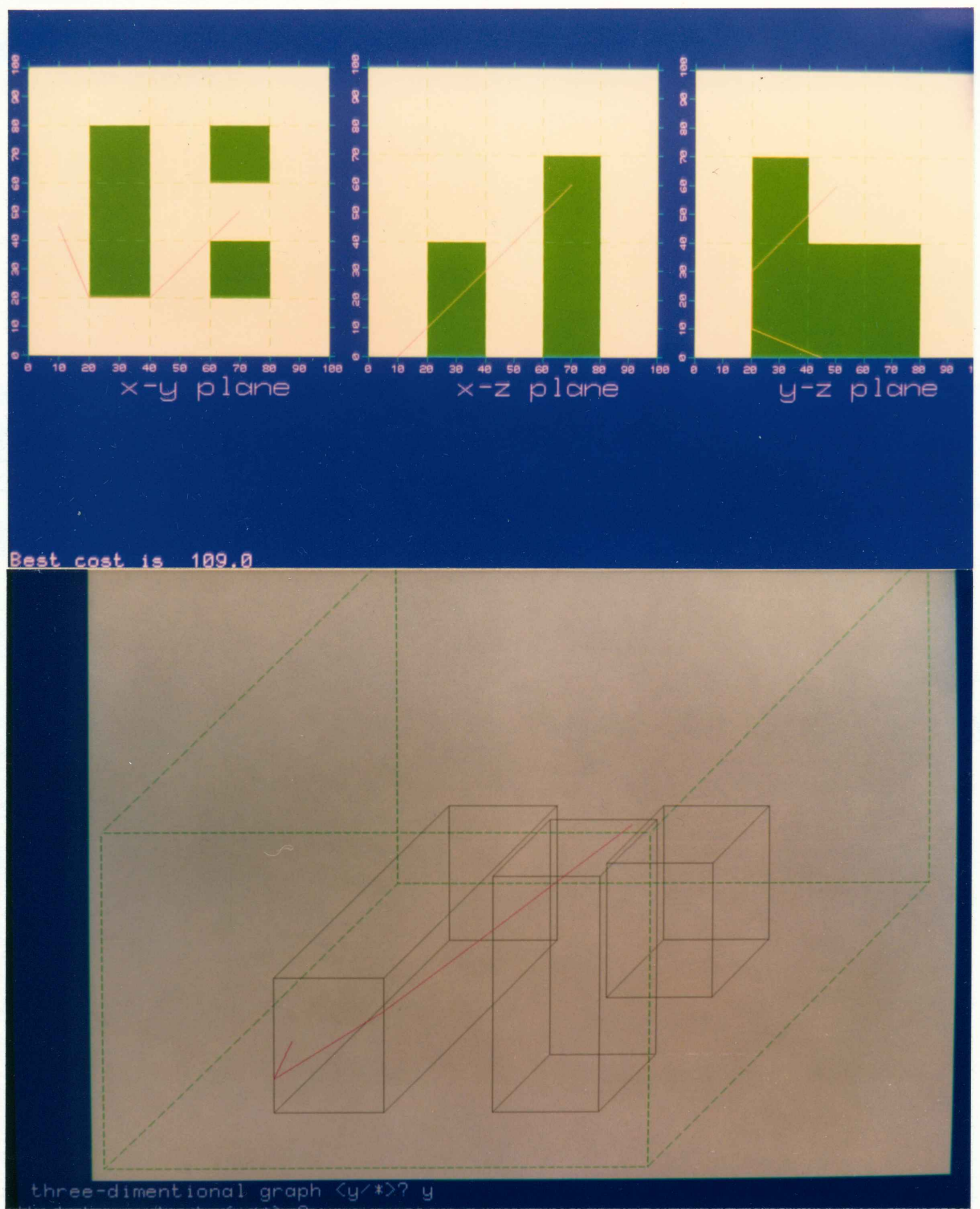

Figure 3-2. Examples of three-dimensional path planning. Three orthogonal two-dimensional projections of the three-dimensional workspace and reconstruction of three-dimensional models and path. 


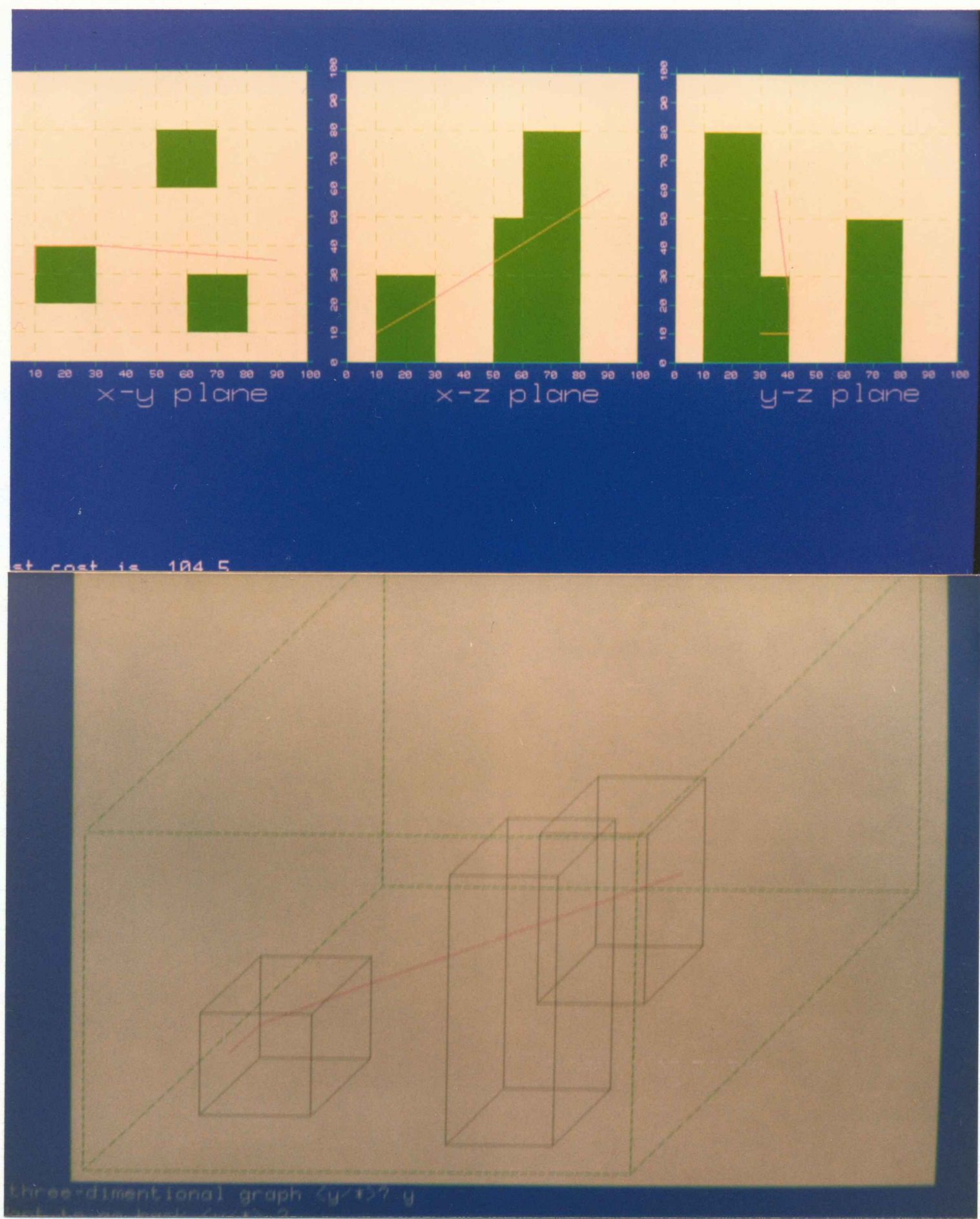

Figure 3-2. ( Examples continued ) 


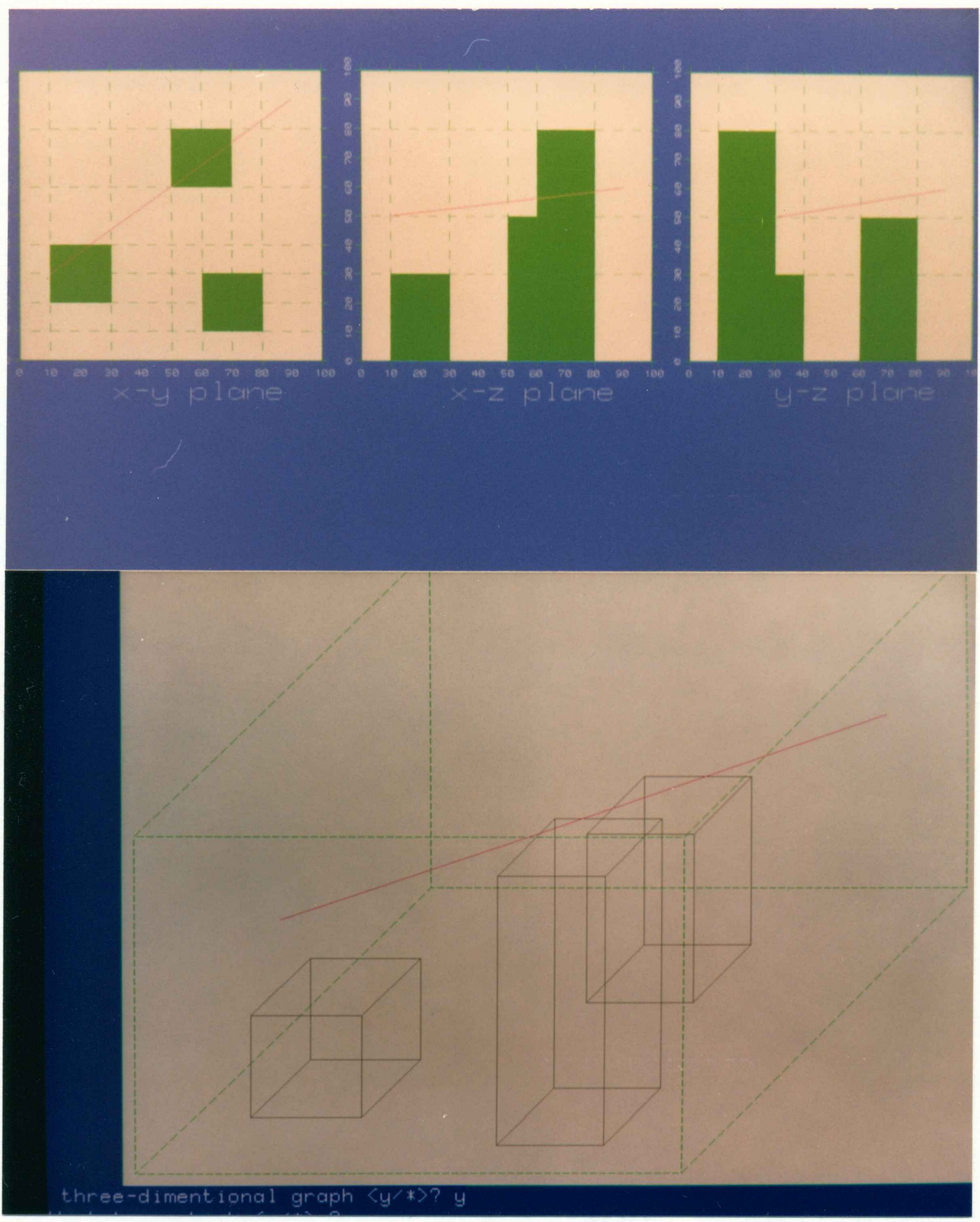

Figure 3-2. ( Examples continued) 


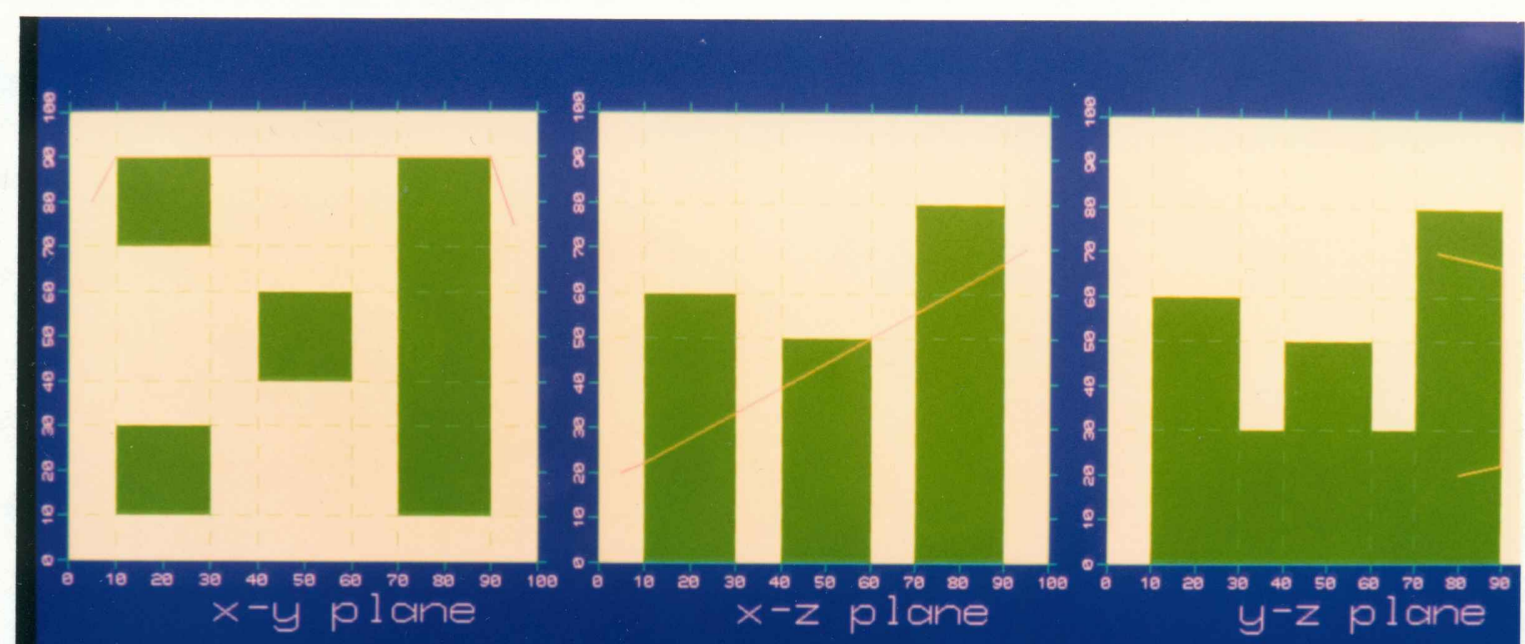

Best cost is 119.1

Figure 3-2. ( Examples continued) 

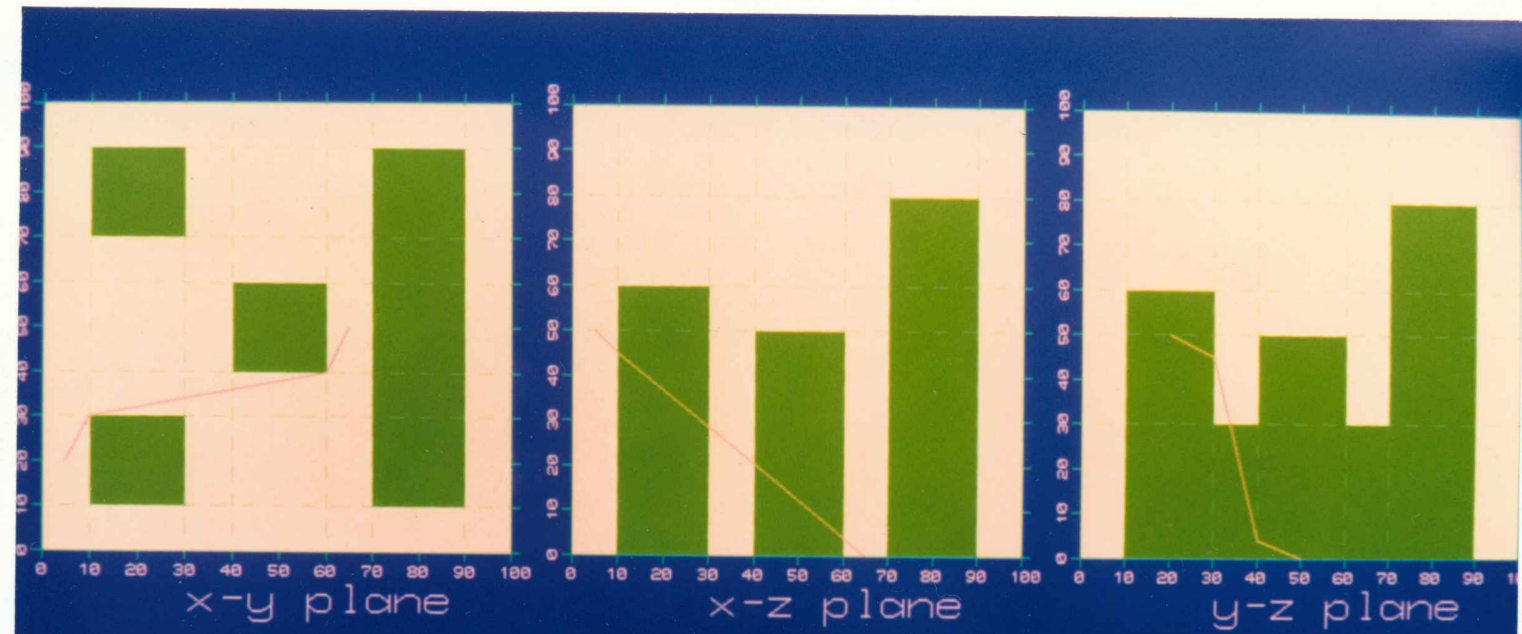

Best cost is 89.7

Figure 3-2. ( Examples continued) 


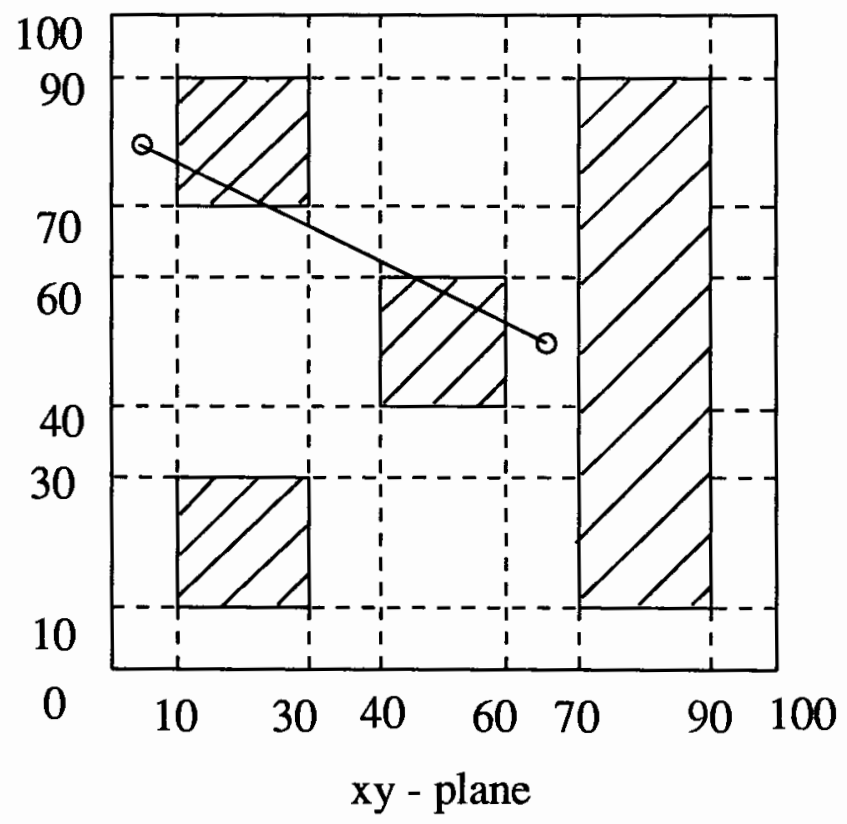

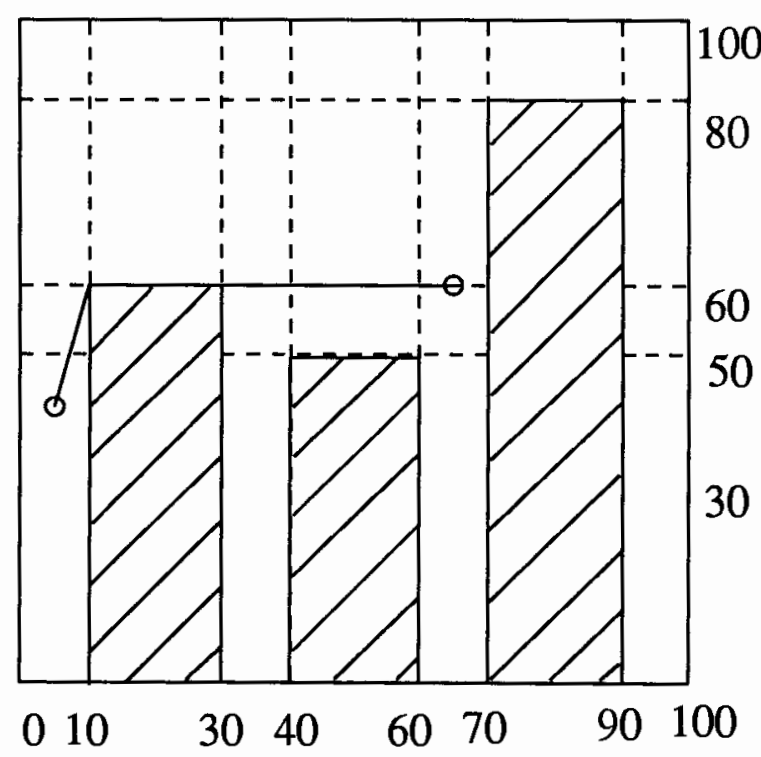

$\mathrm{xz}$ - plane

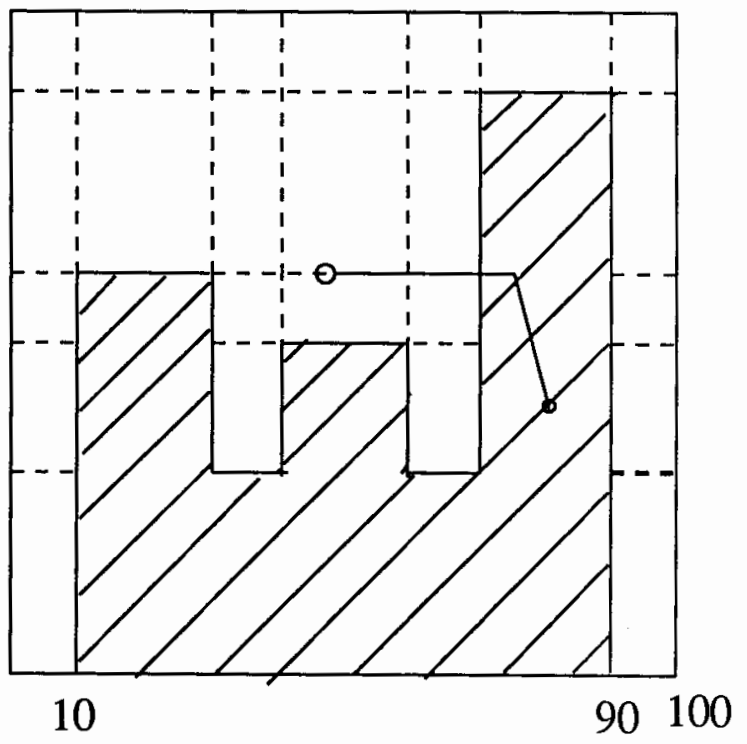

yz - plane

Figure 3-5. Example of the severe occluding effect. (a)-(c) three orthogonal two-dimensional projections of the three-dimensional workspace. 


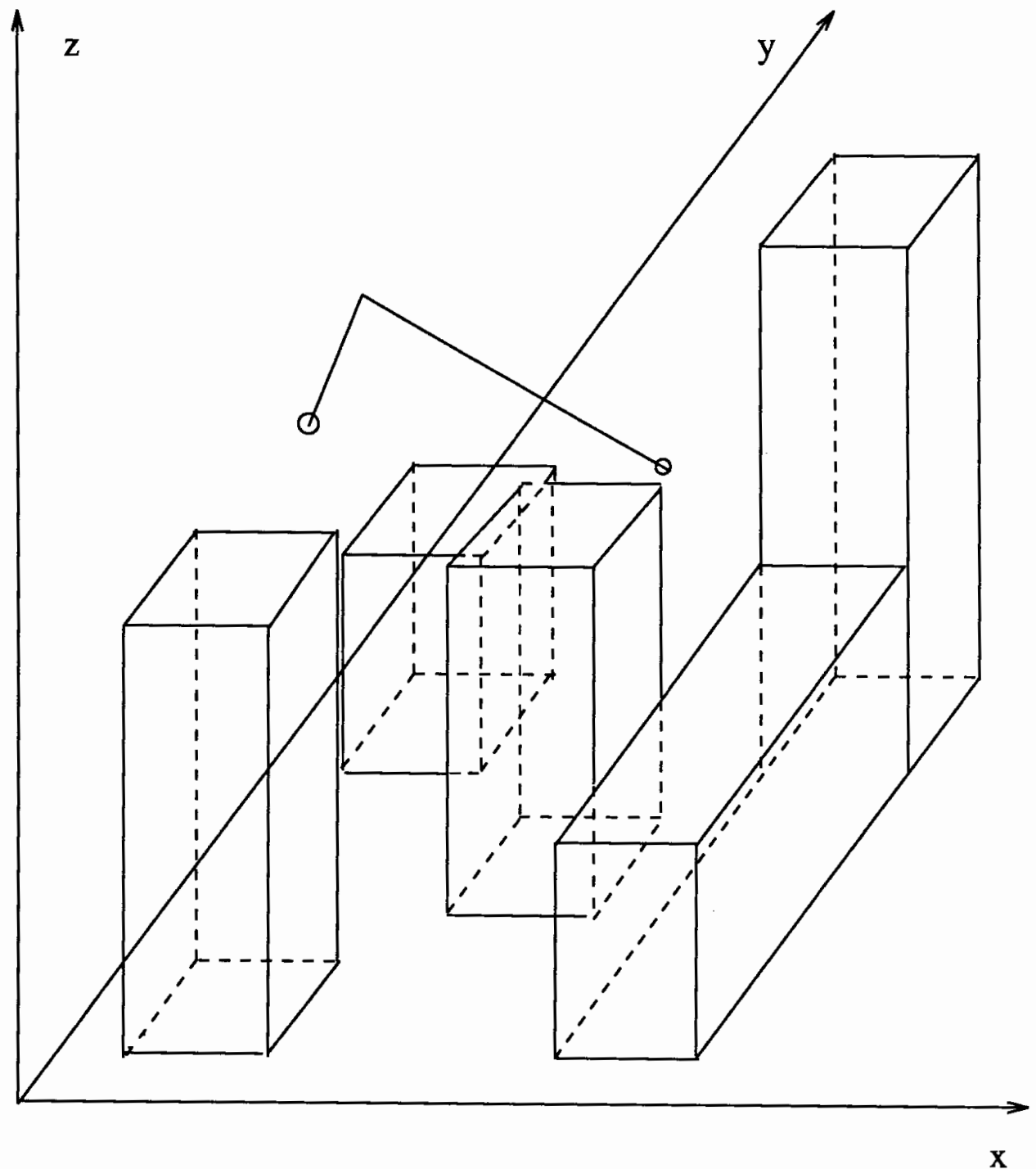

Figure 3-5. ( Continued) (d) reconstruction of three-dimensional models and path. 


\section{CHAPTER IV}

\section{CONCLUSION}

The path planning algorithms for a two-dimensional layout and a threedimensional workspace have been developed in terms of Euclidean distance. The path planning procedure outlined in this paper takes advantage of all the collision free areas in a workspace. This allows for most suboptimal paths made up of straight line segments to be found efficiently. And this suboptimal paths can be modified for near optimal path costs by knowing where the obstacles are positioned. In addition, if the source and the destination points fall inside the same collision free area, then the optimal two-dimensional or three-dimensional path is picked trivially as a straight line path.

In two-dimensional path planning, near optimality is obtained by taking into consideration all the rectangular two-dimensional nodes and by conducting a complete search of graph obtained. The parameter $\beta$ is introduced to systemmetically trade off optimality for execution speed in the backtracking graph traversal algorithm. With the smallest value of $\beta(\beta=1)$, the result is the smallest computational time but the suboptimal paths. Taking advantage of this suboptimal path established and the smallest execution time, this suboptimal path can be modified to be near optimal path without increasing computational time ( average 0.1 second increase ). The speed of the algorithm is governed by both the graph size and the structure. Since the number of graph nodes in a layout containing $\mathbf{n}$ inadmissible areas that is representing obstacles is bounded by $\mathrm{O}\left(\mathrm{n}^{2}\right)$, execution time roughly has the complexity of the same order. 
In the three-dimensional path planning, a set of three orthogonal twodimensional projections is used to represent a three-dimensional workspace. The three-dimensional path planning is decomposed into the problem of twodimensional path planning in the three orthogonal two-dimensional projections. Based upon the two-dimensional path that is chosen among three two-dimensional paths from three orthogonal subspaces, the three-dimensional path is constructed. Once a three-dimensional path is established, a three-dimensional path point can reach other three-dimensional path point (where these two points are not adjacent) by some collision free path segments if the three-dimensional path segments are collision free in one or more of their respective orthogonal subspaces. This procedure is simpler than forming three-dimensional geometric models of the obstacles in the workspace. Because of the limited information obtained from three orthogonal subspaces, a path could not be found even one exists in realty. This problem could be improved by using different (additional) camera viewing angles other than the orthogonal angles. The time complexity depends on the total number of two-dimensional nodes in the three two-dimensional subspaces, which is $O\left(3 n^{2}\right)$.

The time complexity mentioned above is loose upper bounds. If obstacles edges line up at all, as in the case of industrial layout, a much lower bound generally holds and the graph complexity decreases drastically. The number of arcs which represent the intersections of prime convex areas generally decrease if the setup is cluttered with aligned objects. This result in faster search through the graph and consequently speed improvement for the procedure.

No efficient algorithms currently exist for planning optimal paths among three-dimensional obstacles. It is also pointed out that the most important fact for the space representation is to avoid excess detail (and therefore time spent) on 
parts of the space that do not affect the operation. Therefore, the space representation should not have to maintain a perfectly detailed model everywhere. Results presented in this paper are meant to serve as an initial step toward this aim. And there must be some more thought about representation of obstacles. 


\section{APPENDIX A}

\section{SAMPLE LAYOUT}

Another example of a typical layout is presented in figure A-1(a), which has two obstacles. The Areas marked A through $\mathrm{H}$ in (a), (b), and (d) denote all the prime convex areas for this layout in figure A-1(a). In figure A-2, the procedure to obtain all prime convex areas is illustrated. Figure A-3 shows the node graph obtained from the layout of figure A-1(a) and areas of intersection assocoiated with each arc.

The comparision of layout of figure 2-1(a) with that of figure A-1(a) shows that the former has more obstacles but the latter has more prime convex areas. The reason for this is that most of obstacle edges line up as in figure 2-1(a). Then the graph complexity decreases drastically, and consequently the execution time for graph search is faster. 


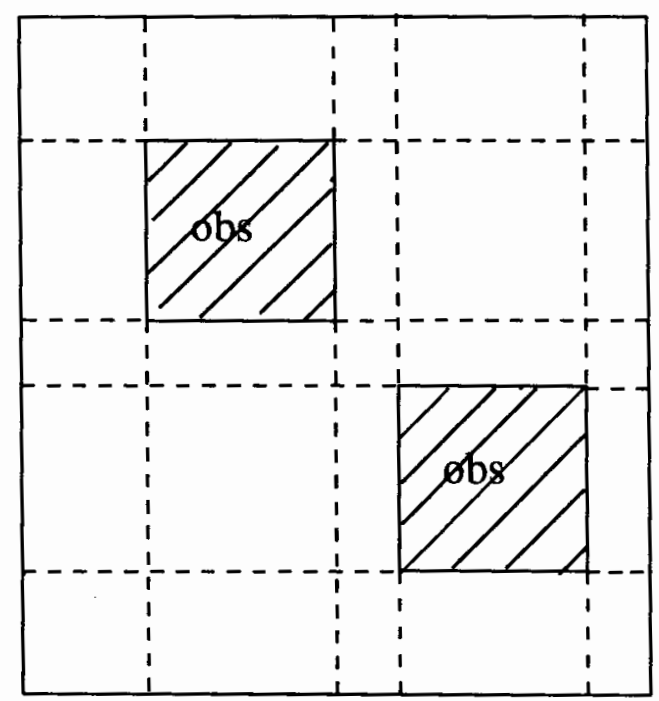

(a)

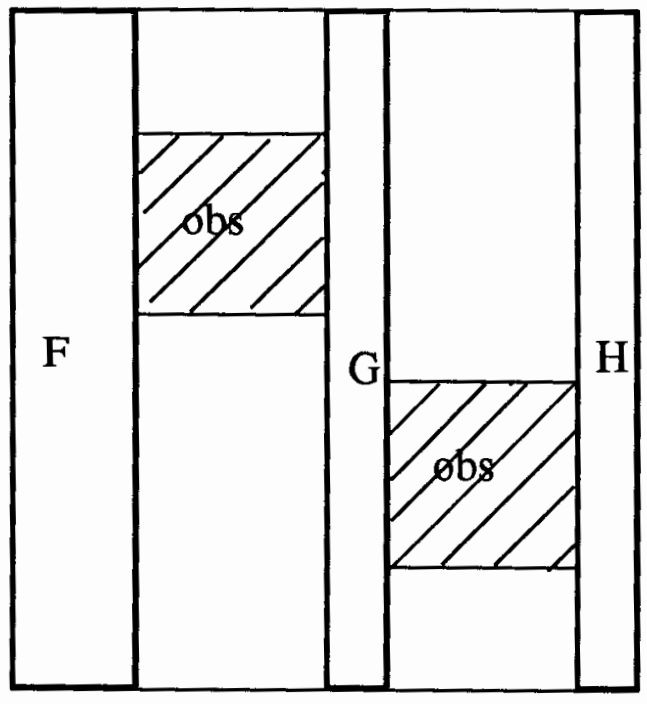

(c)

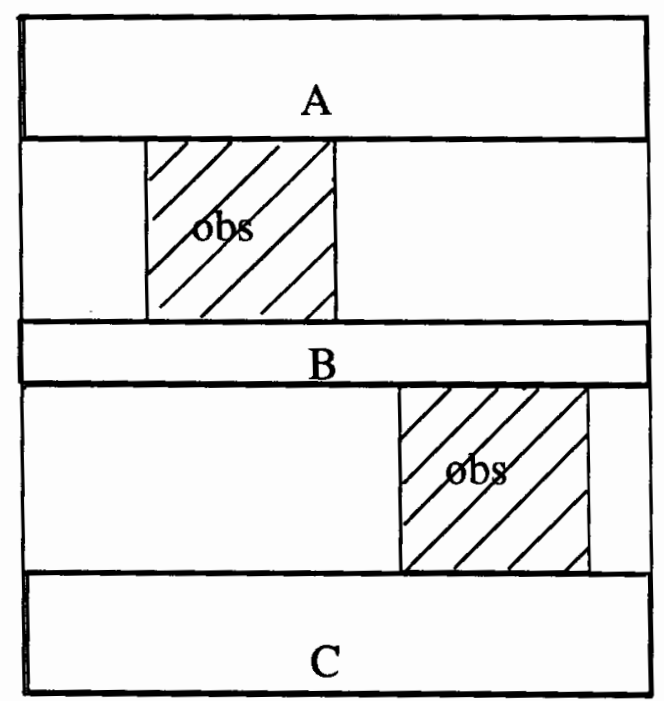

(b)

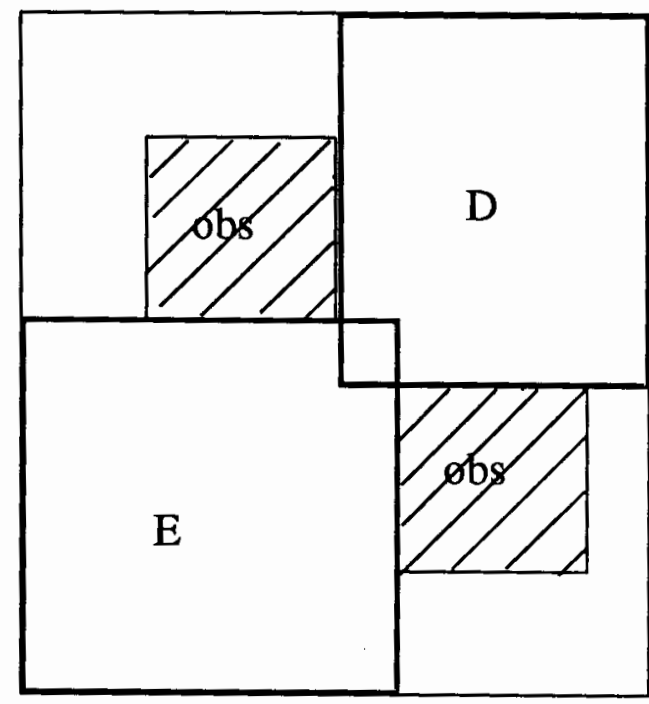

(d)

Figure A-1. Typical layout of two obstacles. Layout is shown in (a) and the areas marked $A$ through $H$ in (b), (c), and (d) present all the prime convex areas in this layout. 
list 1

1111110000

1011101000

1111100100

1110100010

1111100001

list 2

1111110000 (A)

$1000001000(X)$ 0011101000 (X)

1111100100 (B)

$1110000010(\mathrm{X})$

$0000100010(\mathrm{X})$

1111100001 (C)

list 3

$1000011000(\mathrm{X})$

$0011111000(\mathrm{X})$

$1000001100(\mathrm{X})$

0011101100 (X)

$1110000110(\mathrm{X})$

$0000100110(\mathrm{X})$

1110000011 (X)

$0000100011(\mathrm{X})$ list 4

1000011100 (X)

0011111100 (D)

$1000001110(\mathrm{X})$

$0010001110(\mathrm{X})$

0000101110 (X)

1110000111 (E)

0000100111 (X)

list 5

$1000011110(\mathrm{X})$

$0010011110(\mathrm{X})$

$0000111110(\mathrm{X})$

$1000001111(\mathrm{X})$

0010001111 (X)

0000101111 (X)

list 6

1000011111 (F)

$0010011111(\mathrm{G})$

$0000111111(\mathrm{H})$

Figure A-2. The procedure to obtain all prime convex areas for layout of figure A-1(a). The prime convex areas remain unmarked and are labeled as $\mathrm{A}$ through $\mathrm{H}$. 


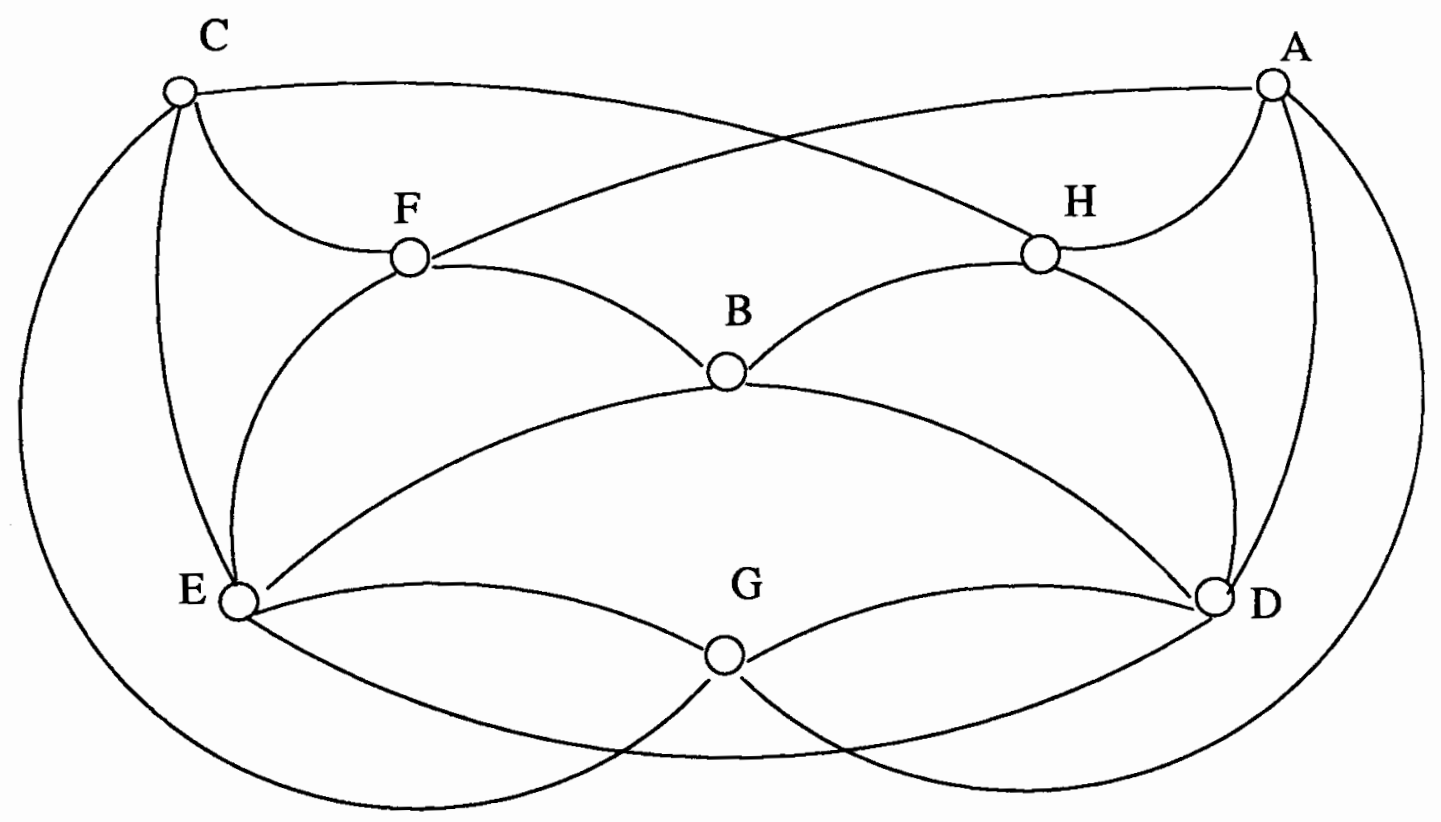

(a)

arc nodes area of intersection

\begin{tabular}{|c|c|c|}
\hline & $A-D$ & 0011110000 \\
\hline & $A-F$ & 1000010000 \\
\hline & $A-G$ & 0010010000 \\
\hline & A - H & 0000110000 \\
\hline & $B-E$ & 1110000100 \\
\hline & B - D & 0011100100 \\
\hline & $B-F$ & 1000000100 \\
\hline & $B-G$ & 0010000100 \\
\hline & B - H & 0000100100 \\
\hline & $C-E$ & 1110000001 \\
\hline & $C-F$ & 1000000001 \\
\hline 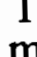 & $C-G$ & 0010000001 \\
\hline & $\mathrm{D}-\mathrm{E}$ & 0010000100 \\
\hline & $E-F$ & 1000000111 \\
\hline & $G-E$ & 0010000111 \\
\hline & $G-D$ & 0010011100 \\
\hline & & 0000111100 \\
\hline
\end{tabular}

(b)

Figure A-3. Graph of intersecting prime convex areas for layout of figure A-1(a) and areas of intersection associated with each arc. 


\section{APPENDIX B \\ TWO DIMENSIONAL PATH PLANNING PROGRAM}

In this appendix, two-dimensional path planning procedure is outlined

horizontal_strip

Represent each horizontal strip such that

if ( prime convex areas )

free areas by 1 's

obstacle areas by 0's

if (obstacle areas )

free areas by 0's

obstacle areas by 1 's

find_node_area

Find all contiguous horizontal strip

Make a list of all strings

Generate a new list of strings

until new list has only one group

Find intersections among nodes

Determine areas occupied by intersections and nodes

find_obstacle_area

Find all contiguous horizontal strip

Make a list of all strings 
Generate a new list of strings until new list has only one group

Determine areas occupied by obstacles

initialize

bestcost $=\infty$.

currentcost $=0$.

unmark all graph arcs.

Set tcosts for all arcs not emanating from a destination node $=\infty$.

Set tcost all arcs emanating from a destination

$$
\text { node }=0 .
$$

findnewnode $\left[\mathrm{X}_{i+1}\right]$

Choose $X_{i+1}$ such that

$$
\begin{aligned}
& \text { an arc exists between } X_{i} \text { and } X_{i+1}, \\
& \text { and } X_{i+1} \text { is not on current node path, } \\
& \text { and } X_{i+1} \text { is not in } S \text {, } \\
& \text { and the arc between } X_{i} \text { and } X_{i+1} \text { is nat marked. }
\end{aligned}
$$

Set backtrackflag true

if one of the destination nodes is reached, or if a new node can not found.

moveforward[to $\left.\mathrm{X}_{i+1}\right]$

Add $X_{i+1}$ to current node path.

Determine $C_{i}$ ( as explained in text $)$.

Add $C_{i}$ to current point path.

Add cost segment $C_{i-1} \rightarrow C_{i}$ to currentcost.

$i=i+1$.

backtrack[from $\mathrm{X}_{i+1}$ to $\mathrm{X}_{i}$ ] 
Unmark all arcs originating from $X_{i+1}$.

Mark ark from $X_{i}$ to $X_{i+1}$.

min $=$ minmum tcost associated with an arc emanating from $X_{i+1}\left(\right.$ except $\left.X_{i+1} \rightarrow X_{i}\right)$.

if $\min <\infty$, then

delete each $\operatorname{arc} \mathrm{X}_{i+1} \rightarrow \mathrm{X}_{j}(i \neq j)$

with tcost $>\beta \times$ min

and set tcost of $X_{i} \rightarrow X_{i+1}=\min +$ costsegment

and if currentcost $<$ bestcost, then

bestcost $=$ currentcost

and node path = current node path.

Reduce the currentcost by the cost segment $\mathrm{C}_{i-1} \rightarrow \mathrm{C}_{i}$.

Remove $X_{i+1}$ from current node path.

Remove $\mathrm{C}_{i}$ from current point path.

$i=i-1$.

path planning (main program)

Choose a working space and

input the name of the file

horizontal_strip

find_node_area

find_obstacle_area

Input source points and

destination points

Determine the source nodes and the destination nodes.

if the source and destination points in same node, then compute straight line path.

else 
initialize.

for every starting node

$i=0$.

backtrackflag $=$ false .

findnewnode $\left[\mathrm{X}_{i+1}\right]$.

while newnode exists or $\mathrm{i}>0$

if backtrackflag = true, then

backtrack $\left[\mathrm{X}_{i+1}\right.$ to $\left.\mathrm{X}_{i}\right]$.

findnewnode $\left[\mathrm{X}_{i+1}\right]$.

end if.

if newnode exists, then

moveforward[to $\left.X_{i+1}\right]$.

if currentcost $>$ bestcost, then

backtrackflag $=$ true.

else

findnewnode $\left[\mathrm{X}_{i+1}\right]$

end if.

end if.

end while.

end for.

Choose node path found in backtracking procedure as a optimal path

Modification 1 ( This modification procedure

Modification 2 is explained in Chapter II )

Modification 3

Modification 1

Modification 1 
end if. 


\section{APPENDIX C \\ THREE DIMENSIONAL \\ PATH PLANNING PROGRAM}

In this appendix, three-dimensional path planning procedure is outlined

horizontal_strip

Represent each horizontal strip such that

if ( prime convex areas )

free areas by 1 's

obstacle areas by 0 's

if (obstacle areas)

free areas by 0's

obstacle areas by 1 's

find_node_area

Find all contiguous horizontal strip

Make a list of all strings

Generate a new list of strings

until new list has only one group

Find intersections among nodes

Determine areas occupied by

intersections and nodes

find_obstacle_area

Find all contiguous horizontal strip

Make a list of all strings 
Generate a new list of strings

until new list has only one group

Determine areas occupied by obstacles

find_case

case $1: S$ and $D$ points exist in $x y, x z$, and $y z$ planes.

find path in each plane.

Construct three-dimensional path in each case.

Choose a optimal path form them.

case $2: S$ and $D$ points exist in $x y$ and $x z$ planes.

find path in each plane.

Construct three-dimensional path in each case.

Choose a optimal path form them.

case $3: S$ and $D$ points exist in xy and yz planes.

find path in each plane.

Construct three-dimensional path in each case.

Choose a optimal path form them.

case $4: S$ and $D$ points exist in xy plane.

find path

Construct three-dimensional path.

initialize initialize

bestcost $=\infty$.

currentcost $=0$.

unmark all graph arcs.

Set tcosts for all arcs not emanating from a

destination node $=\infty$.

Set tcost all arcs emanating from a destination

$$
\text { node }=0 \text {. }
$$


findnewnode $\left[\mathrm{X}_{i+1}\right]$

Choose $\mathrm{X}_{i+1}$ such that

an arc exists between $X_{i}$ and $X_{i+1}$,

and $X_{i+1}$ is not on current node path,

and $X_{i+1}$ is not in $S$,

and the arc between $X_{i}$ and $X_{i+1}$ is nat marked.

Set backtrackflag true

if one of the destination nodes is reached,

or if a new node can not found.

moveforward[to $\left.\mathrm{X}_{i+1}\right]$

Add $X_{i+1}$ to current node path.

Determine $C_{i}$ (as explained in text ).

Add $C_{i}$ to current point path.

Add cost segment $C_{i-1} \rightarrow C_{i}$ to currentcost.

$i=i+1$.

backtrack[from $\mathrm{X}_{i+1}$ to $\left.\mathrm{X}_{i}\right]$

Unmark all arcs originating from $X_{i+1}$.

Mark ark from $X_{i}$ to $X_{i+1}$.

$\min =$ minmum tcost associated with an arc emanating from $X_{i+1}\left(\right.$ except $\left.X_{i+1}>X_{i}\right)$.

if $\min <\infty$, then

delete each $\operatorname{arc} X_{i+1} \rightarrow X_{j}(i \neq j)$

with tcost $>\beta x$ min

and set tcost of $X_{i}>X_{i+1}=\min +$ costsegment

and if currentcost $<$ bestcost, then

bestcost $=$ currentcost

and node path $=$ current node path . 
Reduce the currentcost by the cost segment $\mathrm{C}_{i-1} \rightarrow \mathrm{C}_{i}$.

Remove $X_{i+1}$ from current node path.

Remove $\mathrm{C}_{i}$ from current point path.

$i=i-1$.

find path

initialize.

for every starting node

$$
i=0 \text {. }
$$

backtrackflag $=$ false .

findnewnode $\left[\mathrm{X}_{i+1}\right]$.

while newnode exists or $\mathrm{i}>0$

if backtrackflag = true, then

backtrack $\left[\mathrm{X}_{i+1}\right.$ to $\left.\mathrm{X}_{i}\right]$.

findnewnode $\left[\mathrm{X}_{i+1}\right]$.

end if.

if newnode exists, then

moveforward[to $\left.\mathrm{X}_{i+1}\right]$.

if currentcost $>$ bestcost, then

backtrackflag $=$ true.

else

$$
\text { findnewnode }\left[\mathrm{X}_{i+1}\right]
$$

end if.

end if.

end while.

end for.

Choose node path found in backtracking

procedure as a optimal path 
Modification 1 (This modification procedure

Modification 2 is explained in Chapter II )

Modification 3

Modification 1

Modification 1

path planning (main program)

Choose a three-dimensional working space and input the name of the file for $x y, x z$, and $y z$ plane horizontal_strip find_node_area find_obstacle_area

end for. Input source points and destination points

Determine the source nodes and the destination nodes.

if the source and destination points in same node in any two-dimensional projection, then compute straight line path.

else

find_case

Modification

This start by using each point in the three-dimensional point path. Extension of modification 1 to three orthogonal subspaces.

end if. 


\section{REFERENCES}

1. R. A. Brooks

"Planning collision-free motions for pick-and-place operation", Int. J. Robotics Res., vol. 2, no.4, Winter 1983.

2. R. A. Brooks

"Solving the find path problem by good representation of free space", IEEE Trans. Syst., Man Cybern., vol. SMC-13, pp. 190-197, Mar. 1983.

3. E. W. Dijkstra

"A note on two problems in connection with graph", Numerische Mathmatik, vol. 1, pp. 269-271, 1959.

4. G. M. Hunter and K. Steiglitz

"Linear transformation of pictures represented by quad trees", Comp. Graphics and Image Processing, vol. 10, pp. 289-296, 1979.

5. G. M. Hunter and K. Steiglitz

"Operation on images using quad trees", IEEE Trans. Patt. Anal. Mach. Intell., vol. PAMI-I, no. 2, Apr.1979.

6. T. Lozano-Perez

"Spatial planning: a configuration space approach", IEEE Trans. Computers, vol. C-32, no. 2, Feb. 1983.

7. T. Lozano-Perez

"Automatic planning of manipulator transfer movements", IEEE Transactions Syst., Man, Cybern., vol SMC-11, no. 10, Oct. 1981.

8. T. Lozano-Perez and M. A. Wesley

" An algorithm for planning collision-free paths among obstacles", Commun. ACM, vol. 22, pp. 560-570, Oct. 1979.

9. A. Martelli

"On the complexity of admissible search algorithms", Artificial Intelligence 8, pp. 1-13, 1977.

10. E. J. McCluskey, Jr.

"Minimization of Boolean functions", Bell Syst. Tech. J., vol 35, pp. 1417-1444, Nov. 1956.

11. N. J. Nilsson

Problem-solving Methods in Artificial Intelligence. New York:McGrawHill,1971. 
12. W. V. Quine

"A way to simplify truth functions", Amer. Math. Monthly, vol. 62, pp. 627-631, Nov. 1955.

13. J. S. Singh and M. D. Wagh

"Robot path planning using intersecting convex shapes: Analysis and Simulation", IEEE Journal of Robotics and Automation, vol. RA-3, NO. 2, pp. 101-108, Apr. 1987.

14. S. Udupa

"Collision detection and avoidance in computer manipulators", 5th Int. Joint Conf. Artificial Intelligence, Cambridge, MA: Massachusetts Institute of Tech., 1977.

15. E. K. Wong and K. S. Fu

"A hierarchical orthogonal space approach to three-dimensional path planning", IEEE Journal of Robot and Automation, vol. RA-2, NO. 1, pp. 4253, Mar. 1986. 